$$
\text { DOE/ER/4065Y } 3
$$

\title{
HARVARD UNIVERSITY HIGH ENERGY PHYSICS
}

\section{Grant DE-FG02-91ER40654}

\section{Overview}

The principal goals of the work described here are to carry out forefront programs in high energy physics research and to provide first rate educational opportunities for students. Our experimental program is carried out at the major accelerator centers in the world and addresses some of the most important questions in high energy physics. The program is based at Harvard's High Energy Physics Laboratory (HEPL), which has offices, computing facilities, and engineering support, and both electronics and machine shops. Our educational efforts are concentrated in graduate education, where we are currently supporting twelve research students. In addition, undergraduate students work on projects at HEPL during the academic year and over summers.

The faculty group leading the HEP research program at Harvard includes:

Professor Gary Feldman is chair of our High Energy Physics Committee. Since his arrival at Harvard in 1990 he has led the effort planning for the SDC experiment at the SSC and has also worked on CDF. Together with Sanjib Mishra he is now starting a program in neutrino physics. He is a Divisional Associate Editor of Physical Review Letters and has recently completed terms both on HEPAP and as Chairman of the Division of Particles and Fields.

Dr. George Brandenburg is a Senior Research Fellow and the Director of the High Energy Physics Laboratory. He has co-led the the CDF group since he came to Harvard in 1980. He is the local host for the U.S. Particle Accelerator Summer School which is being held at Harvard this summer. He is also serving on the Federal Networking Council Advisory Committee which is advising DOE, NSF and other agencies on computer networking.

Professor Melissa Franklin has been a co-leader of the CDF group since she joined the faculty four years ago and has been active on the experiment for eight years. She received tenure from Harvard this past summer and we look forward to her continuing leadership in future years. She currently serves on the APS Committee on Women in Physics.

Professor Roy Glauber is the head of the theory group which is also supported under this grant. His work includes studies of diffractive scattering processes and many other topics.

DISTRIBUTION OF THIS noriument is ismimitas

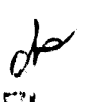


Professor John Huth will be joining our program as a tenured member this July. His research has been on the CDF experiment since he joined the Fermilab staff about ten years ago. After his arrival he will co-lead the CDF group and participate in the SDC program. He has just been appointed to the Fermilab PAC.

Associate Professor Kay Kinoshita has been one of the co-leaders and a key member of the CLEO group since 1985. She has been on research leave during 1992-93 and will be leaving for a new position in the summer of 1993.

Assistant Professor Sanjib Mishra joined us two years ago and together with Gary Feldman is now starting a program in neutrino physics with the NOMAD collaboration at CERN. He has received a DOE Outstanding Junior Investigator award to carry out this work beginning in summer 1992. He will be on research leave in the spring of 1994.

Professor Karl Strauch has led the Harvard's L3 group since the beginning of the experiment. He has served on numerous committees in the HEP community, and most recently was chairman of the IUPAP Commission on Particles and Fields. He will be retiring from the faculty in the summer of 1993, but plans to continue his work on L3.

Professor Richard Wilson has co-led with Frank Pipkin both the CLEO and the E665 groups since the inception of these experiments. Along with Frank and Karl Strauch he was one of the charter members of the HEP program at Harvard. He currently serves on a number of committees concerned with nuclear safety and world-wide environmental issues.

Assistant Professor Hitoshi Yamamoto joined the faculty three years ago as one of the coleaders of the CLEO group. His special interest has been the development of B-factories for the study of $\mathrm{CP}$ violation, and has received a DOE Outstanding Junior Investigator Award for this work beginning in summer 1992.

In addition to the faculty group responsible for our program are seven postdoctoral research associates: Drs. Konigsberg, and Schlabach with the CDF group, Dr. Hurst with the NOMAD and SDC groups, Drs. Cinabro and Henderson with the CLEO group, Dr. Fang with the E665 group and Dr. Kumar with the L3 group. We wish to hire four new postdoctoral fellows in the coming year. Two of these would work on the brand-new NOMAD project, while the other two would strengthen our ongoing CDF and CLEO efforts. This will be the last year for postdoctoral positions on E665 and L3. 
The High Energy Physics Laboratory facilities are available to all the groups on an equal basis. Our engineers and shops are capable of designing and building state of the art detector systems and their associated readout electronics. We have produced both prototypes for detector R\&D as well as complete major systems for all of our groups. Recent examples include the TOF system for CLEO, the Forward EM Calorimeter for CDF, drift chambers and hodoscopes for E665, parts of the L3 Muon system, and a full length SDC muon chamber module prototype. Our current projects are the design and construction of the Central Muon Extension for CDF, engineering of a new beampipe for the Interaction Region for CESR phase II, and prototype muon drift tube production for SDC. In addition to engineering and shops, HEPL has a VAX workstation cluster which is available to all the groups for data analysis.

The HEPL engineering staff consists of two electronics engineers, a mechanical engineer, a machine shop foreman, and a computer system manager. The technical staff consists of three electronics technicians, a machinist, and two mechanical technicians. The administrative staff consists of the Director, a secretary and a bookkeeper.

The budget projections for 1994 and the two following years are included in tables at the end of this document. (Our budget year runs from November of the previous calendar year through October.) Budget year 1994 is the third year of a five year grant. The operations budget for each of our groups consists of salaries plus a reasonable estimate of the M\&S funds required for the effective functioning of the group, in particular their travel to and from their experiments. Approximately $80 \%$ of the cost of the HEPL facility is covered by the experimental groups - each group's share is in direct proportion to their total budget. The remaining cost of the facility is covered through other sources including funding for detector R\&D and production. Finally, the OJI grants received by Professors Yamamoto and Mishra have been used to start new initiatives which have now become key aspects of our program.

Harvard financially supports our high energy physics research program in many ways. The University pays the full salary of tenured faculty for the academic year from non-contract funds as well as the partial salary of the HEPL Director. It has also provided startup funds and support for special projects, and as of July 1991 has agreed to pay the full academic salary for new junior faculty appointments. The University provides a tuition scholarship for first and second year graduate students. Finally, the High Energy Comnuttee has negotiated with the Dean of the Faculty of Arts and Sciences a special reduced overhead rate (currently $42 \%$ ), which reflects the unique needs of our program. 


\section{Proton-Antiproton Colliding Beam Program at Fermilab - CDF}

(Professors Melissa Franklin, and John Huth; Drs. George Brandenburg, Peter Hurst, Jacobo Konigsberg, Phil Schlabach and Jorge Troconiz; Tom Baumann, Andrew Gordon, Rowan Hamilton, Colin Jessop, David Kestenbaum, George Michail, and Fotis Ptohos)

Since 1980 the primary physics activity of this group has been focused on the CDF program at the Fermilab Tevatron Collider. Our students and postdocs have been in an excellent position to exploit the tremendous physics opportunities provided by $1.8 \mathrm{TeV}$ protonantiproton collisions at the Tevatron. We are producing and studying the properties of the weak vector bosons, searching for new kinds of heavy quarks and leptons, in particular the top quark, and making systematic studies to test the theory of strong interactions, QCD. We are in a position to look for entirely new phenomena such as super-symmetric particles, technicolor, and all manner of other exotic possibilities. Finally the addition of a silicon vertex detector to $\mathrm{CDF}$ has opened a whole range of $\mathrm{b}$ quark physics topics to be investigated.

The leaders of this group, George Brandenburg and Melissa Franklin, will be joined by John Huth of Fermilab, who will be joining the Harvard faculty and the CDF group in July 1993. Other faculty members who have worked with this group in previous years are Roy Schwitters, Marjorie Shapiro, Craig Blocker, and Steve Geer who are now at the SSC Laboratory, U.C. Berkeley, SSCL, and Fermilab respectively.

Our group has also included three talented postdoctoral research associates, Jacobo Konigsberg, Phil Schlabach, and Peter Hurst. Dr. Hurst, who has worked half-time on CDF and half-time on the development of muon systems for the SSC/SDC collaboration, has now joined the new NOMAD group (and remains half-time on SDC). We hope to hire a new postdoc to replace him sometime this fall. We are also fortunate to have a guest postdoctoral fellow from Spain, Jorge Troconiz, during 1993-4. All of our research associates work closely with our graduate students on physics studies such as the search for the top quark. There are currently seven graduate students in the group, and seven have already finished their theses.

The final design for CDF has a central magnetic spectrometer built around a solenoid magnet with charged particle tracking, shower counters, and hadron calorimetry. The Harvard group constructed (together with Brandeis) and is responsible for the electromagnetic shower calorimeter (FEM) for the forward angle regions $\left(2^{\circ}-10^{\circ}\right)$ at both ends of the detector. We 
also have recently produced and installed rings of drift tubes (CMX) at both ends of the CDF detector to extend the muon coverage of the central region from $\eta=0.6$ to $\eta=1.0$.

CDF had a short test run in the fall of 1985 and the first engineering run in the spring of 1987. A long physics run, which began in June 1988 and ended in May 1989, yielded a total integrated luminosity of $4.5 \mathrm{pb}^{-1}$ logged to tape. In our current run, which ends this summer, we will gain a factor of approximately 5 in total integrated luminosity. This will be followed by a run starting in the fall of 1993 which should accumulate over $100 \mathrm{pb}^{-1}$. The next CDF run will begin sometime around 1996.

The commitments of the Harvard group to CDF include: A) the maintenance and support of the Forward/Backward Electromagnetic Calorimeter (FEM), B) the fabrication, installation and commissioning of drift tubes for the Central Muon Extension (CMX), C) the coordination of the "gas" calorimeter systems of CDF including their calibration and the development of new electronics, D) the development and testing of new front-end electronics for the 1996 CDF run, E) developing software for data reduction of the CDF calorimetry and for general purpose use by online and offline analysis programs, and F) participation in the physics analysis and coordination of the Electroweak Physics group. These commitments are described below.

\section{A. Forward/Backward Electromagnetic Calorimeters - FEM}

Our group in collaboration with the Brandeis University group designed, constructed, tested, and installed a large electromagnetic shower counter system for use in the small angle region $\left(2^{\circ}-10^{\circ}\right)$ of the CDF colliding beam detector at Fermilab. The system uses proportional tube calorimetry with lead sheets as the radiator. The proportional tube planes are constructed using a novel technique which combines aluminum tubes and cathode pad readout. The total system (both forward and backward regions) has approximately 3000 independent pad towers which are read out in two depth segmentations.

The FEM calorimeter continues to perform well. Data from the forward caiorimeters are being used in the measurement of the jet scattering angles and in the calculation of the missing transverse energy. Electromagnetic clusters in the forward calorimeters are also being used in the study of the $Z^{\circ}$ vector boson. For the 1992-3 run Dr. Konigsberg has been in charge of recalibrating and commissioning the FEM. 


\section{B. Central Muon Extension - CMX}

It has been clear that good lepton identification capability has been extremely important for many important CDF physics topics, in particular the search for the top quark and the study of $B$ decays. Although there is the potential for electron identification at all angles currently', muons can only be directly identified in the central region, $\theta>55^{\circ}$, and in the forward region, $2^{\circ}<\theta<10^{\circ}$. In particular our group has designed and built an extension of the central muon detector. This utilizes the steel in the yoke and hadron calorimeters to extend the existing coverage from $\theta \approx 55^{\circ}$ down to within $40^{\circ}$ of the beam. It is being accomplished by adding a ring of drift tubes covering the region $40^{\circ}<\theta<55^{\circ}$. The ring has four layers at both ends of the detector, and each ring will contain approximately 1000 drift tubes.

The CMX drift tubes, which were developed at Harvard by Dr. Brandenburg and others, have been manufactured and tested at HEPL and shipped to Fermilab for installation on the CDF detector. At Fermilab Dr. Schlabach has been in charge of the installation and commissioning of the CMX system. The upper three quarters of the system have been installed and are now a functioning part of CDF providing Z's, W's, W's, and hopefully eventual evidence for the existence of the top quark.

\section{Gas Calorimetry and Electronics Development}

Prof. Franklin has been the coordinator of all work on CDF gas calorimetry. This includes four calorimeters covering the plug and forward regions $\left(30^{\circ}>\theta>2^{\circ}\right)$. These systems have been recalibrated with a shorter integration time and outfitted with new electronics to enable the calorimeters to run successfully through the 1991-1994 period, or up to a luminosity of $5 \times 10^{31}$ with 6 bunches. The Harvard group has rewritten the electron reconstruction code in the plug region to give a factor of three more rejection from jets and has made it possible to trigger on plug electrons of $15 \mathrm{GeV}$ and higher. This has extended our $\mathrm{W}, \mathrm{Z}$, and top coverage by $30 \%$

Harvard has made numerous contributions to the front-end electronics for the gas-tube calorimetry over the years. In addition to recently designing new input buffers, these have included the original multi-channel amplifier/integrator boards, anode-pulsing boards, the master clock fan-out system, and others. 


\section{Front-End Electronics Upgrades}

Three fundamental analyses that will benefit from increased luminosity are: a) the top quark search $b$ ) a precision measurement of the $W$ mass and $c$ ) b physics. These will require a substantial increase in luminosity, which is expected to come from accelerator upgrades, including larger number of bunches (132 nsec crossing frequency), the Main Injector and improvements to the pbar source and linac. In order to do the best possible physics up to luminosities of $10^{32}$, it is necessary to make a significant upgrade to CDF electronics. Prof. Huth is presently a project coordinator for the CDF electronics upgrade.

CDF is proposing to move to a digital, pipelined readout scheme. Trigger decisions would be based on digital calorimeter readout with double buffers for two levels of trigger decision. In effect, this scheme allows for a deadtimeless readout system up to the full luminosities expected from the Tevatron. This scheme would preserve the livetime needed for high $p_{t}$ physics (e.g. top search) while allowing for a sufficient bandwidth to get samples of b decay on the path to CP violation tests. It is anticipated that Harvard will play a substantial role in this upgrade, involving the electronics shop at HEPL, and a new postdoc hire.

\section{E. Software Development}

The Harvard CDF group has played a very significant role in the ongoing development and management of the enormous body of code used in analyzing CDF data. The group made significant contributions to the definition of the data structures used for offline analysis. A macro expander used to maintain site dependent code within a single source file was entirely designed and implemented at Harvard. The main analysis driver, used for online calibration and offline reconstruction and physics analysis, was also written at Harvard. Finally, we have developed and now help maintain the version of the CDF event display program, DF, which runs on interactive workstations.

Several members of the Harvard group have been involved in CDF efforts to define the standard CDF reconstruction package. A large portion of the calorimetry reconstruction code has been written and is maintained by our group. Major contributions have been made recently to the electron, muon, and tau reconstruction algorithms. Members of our group have also been involved writing and maintaining the code used online to monitor the quality of the data being written to tape and in the development of a extensively used fast detector simulation package. 


\section{F. Physics Analysis}

After the last run the Harvard group was involved in various electroweak studies and finished a set of measurements of the $\mathrm{Z}$ boson properties: the total cross-section, the forwardbackward asymmetry, and the transverse momentum distribution. The students who completed theses on these topics were Ed Kearns, Peter Hurst and Johnny $\mathrm{Ng}$. In addition William Trischuk completed his thesis on the W mass in the ev channel. (Our postdoc Phil Schlabach did his thesis on the $\mu v$ channel at Univ. of Illinois.) Also a search for a light Higgs boson was done by Steve Geer and others. Prior to the last run the Harvard group contributed primarily to the study of $Q C D$ via the production of high transverse momentum jets. David Brown, Robert Carey, and Richard St. Denis completed their theses in this area.

Finding the top quark is a major goal of the CDF collaboration. For the data from the current run we are actively working on top quark analysis methods in several different channels. Jaco Konigsberg and a student, Tom Baumann, are working on decays involving $\tau$ 's. (Colin Jessop is writing his thesis on a search for top decay to $\tau$ 's via Higgs bosons using last run's data.) Phil Schlabach is pursuing the $\mu$ plus jets channel using the extended muon coverage of the CMX. Fotis Ptohos is writing his thesis on the $\mathrm{W}$ plus jets channel including the plug calorimeter region.

The low-x structure of the proton is of fundamental interest to QCD practitioners. There are a number of excellent handles to extract both the gluon and sea parton distribution functions. One critical measurement is the extraction of a direct charm signal in the the process charm + glue $\rightarrow$ photon + charm, which gives unique data on the charm sea distribution. One mode for a direct charm signal is in gamma-muon events. Jorge Troconiz and Rowan Hamilton are involved in a measurement of the cross section for charm-gamma production.

We also will continue to be involved in electroweak studies: a new student, Andrew Gordon, is planning to do his thesis on the W mass measurement.

A large part of the analysis described above has been performed at Harvard using the HEPL VAX system. Our ability to perform CPU-intensive analysis at our home institution has meant that our graduate students can reside in the Boston area while they are working on their thesis analysis. This fact has allowed us to profitably interact with other members of the Harvard Physics department (both theorists and other experimentalists). This system is now a cluster of powerful workstations (including six VAXstation 4000's) plus approximately 20 gbytes of disk storage and six cassette tape drives. We also have several VAXstations and 
disks at Fermilab. In the coming year we would like to add at least two Alpha workstations to the HEPL cluster and request $\$ 25 \mathrm{~K}$ in equipment funds to purchase these systems. Matching funds for this purchase will be provided by the University for the startup of Professors Franklin and Huth.

\section{G. Timetable}

1993 The first phase of the current CDF run is underway with our group having taken part in the commissioning of both the muon upgrade and the gas calorimetry. We are actively looking at the new data which will total $25 \mathrm{pb}^{-1}$. The second phase of the current run will get underway in late 1993 after the linac is upgraded. Two students should finish their theses this year, while three new students will start their thesis work.

1994 The second phase of our current run will be completed this year hopefully having accumulated more than $100 \mathrm{pb}^{-1}$, which will make it possible to find the top quark or push the lower limit on its mass close to the theoretical upper limits. It will also be possible to make substantial improvements in other measurements such as the $\mathrm{W}$ mass. In addition to extensive data analysis we will be involved in the planning and building of the front end electronics upgrade for the detector. We will also be assembling the lower quadrant of the CMX system.

1995: We will be heavily involved in analysis of the data again this year. We will also be completing the installation of the CMX system and the upgrading of the front end electronics.

1996 Sometime this year CDF will begin a new run with a substantially upgraded detector. There will be a new plug calorimeter replacing the gas systems, a completed muon upgrade, an upgraded vertex detector, and extensive electronics upgrades. The Tevatron will be operating in multi-bunch mode and will deliver luminosities of $5 \times 10^{31} \mathrm{~cm}^{-2} \mathrm{sec}^{-1}$ or above. This run will probably the last CDF run until after the completion of the Fermilab Main Injector, which will make even higher luminosities possible.

\section{DISCLAIMER}

This report was prepared as an account of work sponsored by an agency of the United States Government. Neither the United States Gevernment nor any agency thereof, nor any of their employees, makes any wartanty, express or implied, or assumes any legal liability or responsibility for the accuracy, corıpleteness, or usefulness of any information, apparatus, product, or process disclosed, or represents that its use would not infringe privately owned rights. Reference herein to any specific commercial product, process, or service by trade name, trademark, manufacturer, or otherwise does not necessarily constitute or imply its endorsement, recommendation, or favoring by the United States Government or any agency thereof. The views and opinions of authors expressed herein do not necessarily state or reflect those of the United States Government or any agency thereof. 


\section{Electron Positron Collisions in the Upsilon Region - CLEO}

(Professors Richard Wilson and Hitoshi Yamamoto; Drs. Stuart Henderson and David Cinabro; Michael Saulnier, Ted Liu and Faye Shen)

\section{A. The Hardware Projects}

\section{1) CLEO-II Barrel TOE system (Yamamoto, Henderson, Saulnier, Shen)}

The barrel time of flight counters for CLEO II were built, and assembled at Harvard and installed by Harvard personnel. It has achieved the best timing resolution among TOF systems of its kind (140 ps on Bhabha events and 150 ps for pions in hadronic events). The Harvard group maintains the counters, electronics, and software. In the near future, the electronics must be upgraded to accommodate the new bunch structure which is necessary for higher luminosity.

The bench test of the same counter has demonstrated a time resolution of $110 \mathrm{ps}$. A possible source of the discrepancy is the time jitter of the RF signal which is used as common stop. We are now developing a fast discriminator for the beam button signal which directly picks up the beam crossing time.

\section{2) R\&D for the Next Generation of Time of Elight System (Yamamoto)}

The efficiency for a good hit for the current system is quite low - about $75 \%$ for two jet events. We have started a R\&D project to develop a time of flight system which has time resolution of $80 \mathrm{ps}$ and good-hit efficiency of $95 \%$. Such device will extend the 2-sigma $K \pi$ separation to $1.7 \mathrm{GeV} / \mathrm{c}$ and provide a valuable kaon identification for flavor tagging and reconstruction of heavy particles, particularly charmed particles.

The higher efficiency is obtained by a finer segmentation, and the better timing resolution is achieved by detecting more photons which have short paths to fast photo multipliers. Main tools are an optical table with a light-tight housing, a $\mathrm{N}_{2}$ /dye laser, and a $4.5 \mathrm{~Hz}$ bandwidth wave form digitizer. With the technologies that have recently become available, the timing technology with photo multiplier can be improved one step further. The same technology is critical for the correlated-time Cerenkov detector which is one of the candidates for high momentum $\mathrm{K} \pi$ separation. We are hoping to hire a research associate who can spend a large fraction of his/her time on this project. 
3) Phase-llinteraction Region (IR) Upgrade (Yamamoto, Cinabro, Henderson)

We have initiated a major effort on the design and construction of the new interaction region for the next CESR upgrade (phase II) and accepted the complete responsibility of design/construction of the actively cooled beam pipe and the design of the masks including the study of beam-induced backgrounds. The actively water cooled beam pipe is made of a doublewall Beryllium cylinder with water flowing between the walls. With $44 \mathrm{cc} / \mathrm{sec}$ of flow at 1.5 psi pressure drop, it keeps the temperature to within \pm 5 degrees for $400 \mathrm{~W}$ of energy deposit. Extensive study on cooling characteristics and erosion/corrosion have been performed at Harvard, and the design is now finalized. The order of the Beryllium section has been placed to BWADC (former Electrofusion Co.) two weeks ahead of schedule.

Dr. Henderson and Dr. Cinabro are now the acknowledged experts on the synchrotron radiation background and beam-gas induced background, respectively. In the HEPAP subpanel review in February $1992^{\circ}$ (the Witherel panel) as well as the PAC review in 1993, Yamamoto reported the progress on the IR upgrade representing the CESR/CLEO collaboration. Because of the expertise obtained thus far we are now asked and have decided to get involved in the IR design of the B-factory. Also at major conferences (the Dallas conference, Fermilab DPF, etc.) we have represented the IR upgrade efforts at CESR. In addition to its importance for CLEO, this work is of vital importance for any future high intensity colliders such as a B factory and the SSC.

\section{4) Silicon Drift Detector R\&\&D (Wilson)}

In prior years we developed prototype electronics to read out silicon drift detectors and tested a prototype detector built by SI of Norway. This work was mostly funded by SSC. We are presently measuring radiation damage on these SI detectors Preliminary indications are that at 80 krads the drift time has increased $10 \%$, and above 40 krads the leakage on the $\mathrm{N}$ junction (collector) increases. This increases the noise in any use, and can hopefully be corrected by better electrode design.

We propose to continue these studies of silicon drift and CCD detectors at a low level to be sure that when the new CLEO detector is built silicon drift detectors are understood well enough to be a possible option. The advantages of silicon drift of low collector capacity and low noise, and of less confusion in a high background, may turn out to be important. Budgeting needs are for an undergraduate assistant and for equipment funds $(\$ 10 \mathrm{~K})$ for our share of an order of high resistivity silicon which will be needed for any future fabrication. 


\section{B. Physics Analysis}

The physics output of the CLEO group continues to grow. At the 1993 Washington APS conference, the CLEO collaboration has presented total of 37 talks in physics analysis only which are listed below. The analyses presented by a member of Harvard group are indicated by * and those with major Harvard contribution by + .

$$
\begin{aligned}
& B \rightarrow D(*) \text {, inclusive } \quad B \rightarrow D(*) n \pi \text {, exclusive } \\
& B \rightarrow D n \pi \text {, exclusive } \quad \text { Factorization Tests } \\
& B^{0} \text { semileptonic, } D^{*} \pi^{+} \text {tags } \quad \text { Color Suppressed B Decays } \\
& \mathrm{B} \rightarrow \text { charmonium } \quad \mathrm{B}^{0} \rightarrow \mathrm{D}^{*}+\mathrm{lV} \\
& * \tau+/ \tau^{0} \text {, partial recon.tags } \quad \tau+/ \tau^{0}, D^{*} l v \\
& \text { B } \rightarrow \text { leptons, inclusive Search for } b \text {->ulv, exclusive } \\
& \mathrm{b} \rightarrow \mathrm{ulv} \text {, inclusive } \quad \mathrm{B} \rightarrow \mathrm{XIV} \text {, exclusive } \\
& \mathrm{B} \rightarrow \mathrm{ulv} \text {, inclusive, hermiticity } \quad \mathrm{B} \rightarrow \mathrm{K} \pi, \pi \pi \\
& \mathrm{B} \rightarrow \mathrm{K}^{*} \pi, \mathrm{K} \rho \quad+\mathrm{B} \rightarrow \tau v \\
& \mathrm{~B} \rightarrow \mathrm{s} \gamma \text {, inclusive } \quad \mathrm{B} \rightarrow \mathrm{s} \gamma \text {, exclusive } \\
& D_{s} \rightarrow \mu v \quad D \rightarrow K * l v, D_{-} s->\phi l v \\
& \Lambda_{c} \rightarrow \Lambda \text { XIV } \quad \Xi^{0} / \Xi^{+}
\end{aligned}
$$

Parity viol. in $\Lambda_{c} \rightarrow \Sigma^{+} \pi \quad$ Parity viol. in $D_{s} \rightarrow \phi \rho$

$\mathrm{D}^{* *}$ production and decay $\quad \mathrm{CP}$-viol. in $\mathrm{D}^{0}$ decays

$D_{s}^{*}-D_{s}$ mass difference $\quad$ *Doubly Cabbibo Suppressed Decay

Energy correl. in $\tau \rightarrow \pi v / \tau \rightarrow \pi v \quad B R(1 S->g g \gamma) /(1 S \rightarrow g g g)$

$\gamma \gamma \rightarrow$ two prong $\quad \gamma \gamma \rightarrow \eta_{\mathrm{c}} \rightarrow \mathrm{K}^{0} \mathrm{~K} \pi$

$\gamma \rightarrow t$ all neutral 
The list is by no means complete and there are many other analyses which were not presented at the conference for various reasons. The measurement of $\mathrm{B}^{\circ}-\mathrm{B}^{0}$ mixing by partial reconstruction (Saulnier and Kinoshita who will leave Harvard in June 1993), and B $\rightarrow \mu \nu$ (Yamamoto) have been completed recently. The on-going analyses by the Harvard group are on $\mathrm{D} \rightarrow \mathrm{K} \pi$ doubly-Cabbibo-suppressed decays (Liu and Yamamoto), $\mathrm{B} \rightarrow \mathrm{D}^{(*)} \mathrm{D}^{(*)}$ (Henderson and Yamamoto), and the lifetime ratio of $\mathrm{B}^{+}$and $\mathrm{B}^{0}$ using the partial reconstruction method (Saulnier and Kinoshita).

Recently the flavor tagging method for CP violation proposed by Yamamoto has attracted considerable attention. The method is applicable to a symmetric collider and utilizes the interaction $\mathrm{e}^{+} \mathrm{e}^{-} \rightarrow \mathrm{B}-\mathrm{B}^{0} \pi^{+}$where the charge of the pion acts as a perfect flavor tag for the neutral B meson. If a theoretical calculation of the yield by Randall et. al. is correct, the method has a statistical power comparable to that of $\mathrm{BB}^{*}$ mode which requires complicated flavor tagging using leptons and kaons.

\section{Personnel and Budget}

In 1980, the Harvard CLEO group had two professors; two assistant professors and three postdoctoral fellows, and two graduate students. By 1987 budgetary constraints forced a decline to two professors, one assistant professor and two postdoctoral fellows. The increasing interest in the physics attracted another assistant professor (Yamamoto) in 1991. However, with the untimely death of Prof. Pipkin in December 1992 and the departure of Prof. Kinoshita this year, we are down to one professor, one assistant professor, two postdocs, and three graduate students, and dangerously close to the critical mass. We would like to add a postdoctoral fellow and a graduate student in the coming year.

Equipment funds will also be needed next year for the following projects.

(1) Phase-II IR upgrade: $\$ 85 \mathrm{~K}$ already funded.

(2) The upgrade of the TOF electronics to accommodate the new bunch structure required for higher intensities $(\$ 25 \mathrm{~K})$.

(3) A DEC workstation for physics analysis (\$15K).

(4) Research into finely-segmented TOF systems and correlated-time Cerenkov detector $(\$ 25 \mathrm{~K})$.

(5) High resistivity silicon for drift detector studies (\$10K). 


\section{Search for $v_{\mu}$ to $v_{\tau}$ Oscillations with the NOMAD Experiment at CERN.}

(Professors Gary Feldman and Sanjib Mishra; Dr. Peter Hurst; Thomas Dignan)

\section{A. Physics}

The NOMAD experiment is primarily a search for $\nu_{\mu}$ to $\nu_{\tau}$ oscillations in a kinematic region in which the $v_{\tau}$ mass would have cosmological significance, that is, it would contribute significantly to the mass of the universe. Recent results have made this possibility more likely. Interpretations of the COBE results indicate that the dark matter of the universe cannot be completely comprised of cold matter - some of it has to be relativistic. There are few candidates for relativistic dark matter. Neutrinos are the most likely, and of the neutrinos, one would expect the $v_{\tau}$ to be the heaviest. It would have to have a mass of the order of $10 \mathrm{eV} / \mathrm{c}^{2}$, for if its mass was smaller, it would not contribute enough to the mass of the universe, and if its mass were larger, it would over-close the universe. Recent results on solar oscillations also give some support for a $v_{\tau}$ mass in this region. Both allowed regions for matter oscillations of $v_{e}$ to $v_{\mu}$ give a $v_{\mu}$ mass of about $10^{-3} \mathrm{eV}$. This result coupled with the see-saw mechanism favored by many grand-unified theories give values of the $v_{\tau}$ mass in the range of interest.

We have no a priori idea of what $\sin ^{2}(2 \theta)$ is likely to be for $\nu_{\tau}$ oscillation. The current limit is about $510^{-3}$. However, the possible indications of $v_{e}$ to $v_{\mu}$ oscillations in the sun gives us hope that values of $\sin ^{2}(2 \theta)$ are in a measurable range.

\section{B. The NOMAD Experiment}

The NOMAD experiment uses kinematics to identify events with $\tau$ leptons from neutrino oscillations from other events. For example, when a $\tau$ lepton decays into a charged lepton and two neutrinos, there is missing transverse momentum in the general direction of the charged lepton. Backgrounds arise from normal charged current events in which apparent missing transverse momentum is generated from hadrons in the events that are either mismeasured or missed by the detector. Neutral current processes can also create backgrounds if a lepton or apparent lepton is produced at large transverse momentum to the hadronic shower. It is thus imperative that the detector be able to measure all particles as well as possible.

The NOMAD experiment was originally designed to have high sensitivity for the electronic decay mode of the $\tau$. The active 3 ton target of the experiment consists of about 150 low-mass drift chambers inside the old UA1 dipole magnet. Downstream of the target is an array of TRDs for electron detection, and a highly segmented lead-glass electromagnetic calorimeter. 
Two meters of iron with two sets of large muon chambers sit behind the electromagnetic calorimeter and are used to identify muons. Recent calculations have shown that NOMAD is also quite sensitive to both muonic and hadronic decays of the $\tau$.

The neutrino beam is a horn-focused beam produced by $450 \mathrm{GeV} \mathrm{SpS}$ protons. The experiment will receive $24 \times 10^{18}$ protons on target during its first two years of running. The NOMAD experiment is designed to over an order of magnitude more sensitivity to $v_{\mu}$ to $v_{\tau}$ oscillations than the present limit on $\sin ^{2} 2 \theta$ of $5 \times 10^{-3}$.

\section{Harvard Contribution}

We are particularly interested in enhancing the basic capabilities of the NOMAD experiment. The sensitivity of the NOMAD experiment is limited by backgrounds that arise from missed or mismeasured neutrals. There are three main sources: (a) neutral hadrons (neutrons or $K_{L} \mathrm{~s}$ ), which generally do not interact in the electromagnetic calorimeter, (b) photons whose energy measurement is obscured by charged particles entering the same electromagnetic calorimeter cell, and (c) photons and neutral hadrons that exit the detector at large angles.

The first two problems can be addressed by the construction of a hadronic calorimeter behind the electromagnetic calorimeter. In the first case, the hadronic calorimeter will detect and measure the energy and direction of the hadron. In the second case, the sum of the electromagnetic and hadronic calorimeter measurements will measure the sum of the photon's and charged hadron's energy. The iron plates for such a calorimeter are already in place. We believe that sufficient scintillator and photomultipliers already exist in Europe for this purpose. Thus, we only have to cut and wrap the scintillator, make light pipes, mounts, and provide readout electronics.

While in principle, the third problem, neutral particles leaving the detector at large angles, could be addressed by completing the hadronic calorimeter to cover the top, bottom, and sides of the detector, this is beyond our present capabilities. However, we can attempt to detect particles escaping to the side, and to identify muons that leave the detector at large angles, by inserting Mark II muon chambers into gaps in the magnet, which were originally placed there to bring out the light guides from the UAl calorimeter. The Mark II chambers are available and the only cost is shipping them from SLAC to CERN, mounting them on the detector, and providing readout electronics.

Finally, we are interested in increasing the number of neutrino interactions by using material upstream of the NOMAD experiment. The NOMAD coil, immediately upstream of the drift chambers, more than doubles the mass of the detector, and we have persuaded the 
collaboration to accept these events in the trigger. Preliminary calculations indicate that many of these events will be analyzable for the muonic decay of the $\tau$. If it proves useful, we may consider adding additional active target material upstream of the NOMAD coil. In any case, future, high-sensitivity experiments will probably require active targets, and we wish to understand the problems in using them.

A separate equipment request will be made for these projects.

\section{Other Physics Opportunities}

The NOMAD experiment will have at least $1.1 \mathrm{M}$ charged current events from the main detector and an additional $1.5 \mathrm{M}$ events from the coil, all of which will be analyzed by "an electronic bubble chamber." We are interested in the additional physics topics that can be addressed in a way that has not been possible previously. We list a few examples below:

(1) The CCFR experiment observed an anomalous number of events with separated vertices. If these events were caused by the production and decay of a new heavy particle, then these decays should be distinctive in the NOMAD detector.

(2) The excellent low energy electron and muon identification coupled with the measurements of the hadronic shower will allow an excellent measure of charm production in neutrino events. This should lead to an excellent determination of the $V_{c d}$ element of the Kobayashi-Maskawa matrix.

(3) Measurements of $\sin ^{2} \theta_{w}$ from the ratio of neutral to charge current events are usually limited by the measurement of the electron neitrino spectrum and the charm production cross section. Thus, a precise measurement of $\sin ^{2} \theta w$ may be possible.

(4) A search for $u$ to $c$ neutral current transitions is possible by searching for charm production in neutral current events. The current limit is $5 \times 10^{-3}$, and NOMAD should be able to extend this limit by an order of magnitude.

(5) A more sensitive study of trident production should be possible. Tridents that consist of a neutrino and two muons can be produced by both virtual $W ' s$ and $Z$ 's, and have a large destructive interference. Tridents that consist of a neutrino, an electron, and a muon can only be produced by a virtual $W$, and should have a larger cross section.

(6) The high precision of the detector should provide a more sensitive search for anomalous signals in like-sign dilepton events. 


\section{E. Schedule}

There will be a test run in the fall of 1993 to test the data acquisition system, and the first data run is scheduled to begin in April 1994 and extend until November 1994. There will be a similar run each year thereafter. The experiment is approved for two years of running now, but there is no downstream pressure on the experiment, so it can continue as long as it is useful to do so.

Since the date run is less than one year away, we are under a great deal of pressure to proceed with our enhancement projects quickly. For this reason, we are seeking funds in the budget to hire two research associates for this experiment this year.

\section{F. Other Considerations}

We committed to an experiment at CERN only after a long consideration of whether this physics could be done in the United States. We reluctantly concluded that it could not be prior to the construction of the SSC or the Fermilab Main Injector. In comparison to the $24 \times 10^{18}$ protons on target that NOMAD will receive, the upcoming Fermilab experiment $E-815$ has been promised $1 \times 10^{18}$ protons on target. In addition, the horn-focused CERN beam delivers 20 times more neutrinos per proton than the Fermilab sign-selected beam (which will be used by E-815 and which sacrifices neutrino flux to gain sign selection). The Fermilab neutrino energies are about a factor of 3 higher, giving a total advantage to the CERN beam over the Fermilab beam of $24 \times 20 / 3=160$.

By participating in this experiment we hope to gain the experience we need to design higher sensitivity experiments, which could be run at either the SSC or the Fermilab Main Injector. 


\section{The Solenoidal Detector Collaboration at the SSC}

(Professor Gary Feldman; Drs. GeorgeBrandenburg, Peter Hurst,and John Oliver; Mr. Kevan Hashemi, Mr. Edward Sadowski)

The Harvard SDC group is focusing on the problem of muon detection in the SDC detector. (The muon system lends itself to being constructed by university groups, while the calorimetry system, needed for electron identification and measurement, will probably be largely built at national laboratories.)

We have formed a close collaboration with groups from Brandeis University and Tufts University to work particularly on the design and production of drift chambers that can be produced economically and that can meet the necessary requirements for precision. We are also taking an active role in the design of the alignment system, which is necessary to take full advantage of the precision of the chambers. We are actively consulting with engineers from Draper Laboratory on this project.

We are also involved with the general design of the system and the electronics coordination. Professor Feldman is chairmen of the SDC Muon Technical Board and is a member of both the SDC Technical and Executive Boards. Dr. Oliver is coordinating the design of the muon system front-end electronics, and our collaborator, Professor Bensinger of Brandeis University is the Muon Subsystem Project Manager.

During the past year we have been collaborating with physicists from the University of Washington and the SSC Laboratory in designing the tooling to produce a tull scale prototype of a muon tower, which should serve as the first tower of the barrel muon system, one of forty in the complete detector. This tower will contain 22 layers of muon drift tubes and be approximately $8 \mathrm{~m}$ long by $8 \mathrm{~m}$ wide. Muon drift tubes will be produced in the Seattle and Boston areas, with half the Boston production at Harvard and half at Tufts University. It is expected that the full SDC muon drift tube production will take place at approximately six sites in the United States, one of which will be Harvard.

Under the leadership of Dr. John Oliver, Harvard is taking a leading role in the development of front-end electronics for the muon chambers, and read-out electronics for a laser ranging alignment system. The laser ranging device measures the distance to a remote object by sensing the round-trip phase difference of an amplitude modulated laser pulse, and will achieve an accuracy of order 100 microns over distances of tens of meters. 
Dr. Hurst, Dr. Oliver, and Mr. Hashemi are collaborating other SDC physicists and engineers in the production at Harvard of a prototype "fencepost" for the SDC alignment system. A fencepost is a series of straight-line monitors composed from LED-lens-detector combinations and proximity sensors. Fenceposts will form the skeleton of the SDC alignment system and will be tied together with the laser ranging measurements.

Most of the funds for the engineering and prototyping work described above are being provided by a grant from the Texas National Research Laboratory Commission and from funds from the SSCL funneled through the SDC collaboration. Some funds from the base program are requested to support scientific personnel not covered by either of these other sources. 


\section{Muon Scattering at FNAL * E665}

(Professor Richard Wilson; Dr. Guang Fang; Ashutosh Kotwal)

Experiment E665, deep inelastic muon-nucleon/nucleus scattering at Fermilab, completed its third and final run in February 1992. The group must now complete analysis of the large quantity of data that has been collected. This report reviews the Harvard group's past and present contributions to the construction of the apparatus, data reduction and analysis of experiment E665.

The two-fold purpose of the experiment was to study hadro-production from muonnucleon scattering and the A-dependence of cross section ratios. Because of the high beam energy $(500 \mathrm{GeV})$ the E665 kinematic range extends to $X_{b j}=2 \times 10^{-5}$ and $W^{2}=900 \mathrm{GeV}^{2}$, far beyond any previous experiment. This overlaps the kinematic range of both HERA and CERN. The apparatus consists of propertional and drift chambers, an electromagnetic calorimeter, Cerenkov counters, a Time-of-Flight system, a streamer chamber ( first run only ), and vertex and forward spectrometer magnets. The beam was commissioned in 1987, and E665 took the following data during the three runs:

$\begin{array}{llll}\text { Bun } & \text { Period } & \text { Targets } & \text { Luminosity } \\ 1 & 1987(-88) & \mathrm{H}_{2}, \mathrm{D}_{2}, \mathrm{Xe} & 4.5 \times 10^{36 / \mathrm{cm}^{2}} \\ 2 & 1990 & \mathrm{H}_{2}, \mathrm{D}, \mathrm{C}, \mathrm{Ca}, \mathrm{Pb} & 2.4 \times 10^{36 / \mathrm{cm}^{2}} \\ 3 & 1991(-92) & \mathrm{H}_{2}, \mathrm{D}_{2} & 10^{36} / \mathrm{cm}^{2}\end{array}$

In comparison, HERA experiments have logged integrated luminosity of $0.03 \times 10^{36} / \mathrm{cm}^{2}$ to date.

Three Harvard students have graduated after completing analysis on Run 1. One student and one Research Associate are concentrating on the data reduction and analysis of the second and third runs. The Harvard group contributed to the design and construction of critical portions of the E665 apparatus between 1979 and 1991. The electromagnetic calorimeter was constructed in collaboration with the University of Maryland. The responsibility for planning, design and construction of the second level trigger electronics was taken over from the University of California at San Diego. During the upgrade of the detector for the 1990/91 runs the Harvard group constructed a hodoscope, a veto counter array and electronics for part of the trigger and beam monitoring systems. Finally, the Harvard group built the electronics for the vertex detector. There is no ongoing participation in construction. 
The data analysis is almost complete for the 1987 run, is in progress for the 1990 run and is beginning for the 1991 run. The Harvard group's major responsibility continues to be calibration and reduction of the calorimeter data. The collaboration has made major efforts to understand scattering at small values of $X_{b j}$ which depends critically on the ability to understand and correct for the dominant muon-electron scattering and muon bremsstrahlung. This relies heavily on the continuing efforts of the Harvard group on the calibration of the calorimeter. Members of the group are also involved in efficiency studies of detectors and Monte Carlo simulation of the apparatus. Efforts in these areas are expected to continue through 1993.

The Ph.D. theses completed by Harvard students on E665 ( using the 1987 data ) are:

D Michael, A study of Transverse Momentum and Jets Using Forward Hadrons and Photons in Deep Inelastic Muon Scattering at $490 \mathrm{GeV}$; graduated April 1990.

M Schmitt, Deep Inelastic Exclusive Rho Zero Production using $485 \mathrm{GeV}$ Muons; graduated October 1991.

J Conrad. A Study of the $Q^{2}$ dependence of the QCD coupling constant from the Transverse Momentum of Jets in Deep Inelastic Muon Scattering; graduated December 1992.

The following publications have been and are being made on E665 (1987 results):

Spectrometer Details (NIM)

Saturation of Shadowing at very low Xbj (PRL)

Shadowing in muon-Xe scattering (Phys. Lett. B)

$\sigma_{\mathfrak{n}} / \sigma_{p}$ at low $X_{b j}$ (Phys. Lett. B)*

Distribution of charged hadrons using Streamer Chamber (Z. Phys.)*

Distribution of forward charged hadrons (Phys. Rev. D)*

Bose-Einstein Correlations (Phys. Lett.)*

Measurement of Jet production rates (PRL)

Measurement of $\alpha_{s}$ by transverse momentum of Jets (PRL)*

full paper on Jets (Phys. Rev. D)*

Hard QCD in DIS (PRL)*

(" submitted or close to submission )

Draft papers on the following topics are expected in the next few months:

$\mathrm{K}^{\circ}$ using Streamer Chamber

Grey Tracks in the Streamer Chamber

Energy-energy correlations

Intrinsic Transverse Momentum of Partons 
In addition to the studies of cross section ratios at very low $X_{b j}$ the calorimeter has also been vital to the studies of jets, because $1 / 3$ of all the jet energy is in the neutral particles. Jet studies have been one of the major efforts at E665 and Harvard group has been fully involved in the these studies. The preliminary work in the thesis of Michael was expanded. Fractional rates of forward multi-jet production have been measured in the muon-proton scattering for the first dime. These rates are higher than the leading order Monte Carlo calculations.

The Jet rates are critically dependent upon the jet definition and threshold parameter selection. We use the JADE jet algorithm, with a threshold parameter $Y_{\text {cut }}$. Some of the ambiguity ( and infrared divergence in leading order QCD calculations) is removed by the trick due to Altarelli by using the average of the square of the transverse momentum of jets. This is dependent on the quark distribution functions (well known), gluon distribution function (less well known), and the strong coupling constant. This enabled us, in a PRL paper in preparation, to measure the strong coupling constant and demonstrate unequivocally that it varies with $\mathrm{Q}^{2}$ ( the first time in a single experiment). This work is being extended by the collaboration to the 1990 and 1991 data. Our preliminary results on jet production from nuclear targets showed that there is more nuclear shadowing for $2+1$ jet events than for $1+1$ jet events, suggesting fewer gluons in nuclei than given by the sum of nucleons.

Work in progress at E665 (mostly on the 1990 and 1991 data ) includes:

A dependence of jet rates

Energy-energy correlations of hadrons

Jet properties in nucleons and nuclei

$n / p$ ratio to very low $X_{b j}$ with improved statistics and systematics

A dependence of shadowing and saturation of shadowing at low $X_{b j}$

Measurements of structure functions at low $X_{b j}$

Average transverse suomentum of jets as a function of $A$

Measurement of intrinsic parton $k_{\mathfrak{l}}$ by azimuthal asymmetry of forward hadrons

Measurement of the intrinsic parton $k_{t}$ by measurement of average $p_{l}$ of jets

$\mathrm{K}^{\circ}, \Lambda^{\circ}$ production rates and nuclear dependence of strangeness production

$\pi^{+} / \pi$ ratio in fast forward hadrons vs. A to study valence quark enhancement

Bose-Einstein effect in nuclei

Temperature of nuclear excitation vs. $X, Q^{2}$ by the study of low energy neutron spectra

Produztion of vector mesons, nuclear effects, polarization effects

Helicity study of phi mesons

Nuclear effects on forwand hadron muitiplicity as a function of $v$

Nuclear effects on transverse momentum of forward hadrons

Charm production 
Dr. Fang is continuing the work of Mr. Schmitt on muoproduction of vector mesons using the 1990 and 1991 data. The goals of this analysis include measurement of the variation of exclusive vector meson production cross section with kinematic variables in nucleon and nuclear targets; the cross section ratio of longitudinally to transversely polarized vector mesons; decay parameters; and the color transparency effect. Evidence of color transparency has been seen in hadron-nucleus scattering, but has not yet been observed in lepton-nucleus scattering. Finally, inclusive vector meson production will also be studied to gain insight on the strange content of the nucleon and the flavor characteristics of hadronization. With the unique E665 kinematic range this work will span the range from where the vector meson dominance model applies to where the quark model is more appropriate. The preliminary results on the cross section ratios of vector meson production from nuclear targets to that from $D_{2}$ target as functions of $Q^{2}, t, v$ and $X_{b j}$ will be presented in the summer conferences.

Our work on cross section ratios at low $\mathrm{X}_{\mathrm{bj}}$ shows that it is possible to make important contributions to the understanding of low $X$ phenomena by an absolute cross section measurement and that the systematic errors are tractable. This will form the thesis of Ashutosh Kotwal. The statistical errors on the muon-nucleon cross section measurement from the run 91 data are expected to be a few percent or better in the region $0.1<Q^{2}<10$ for $0.0001<X_{b j}<0.08$. The data in the region $0.0001<X_{b j}<0.006,5>Q^{2}>0.1$ is unique since no muon-nucleon cross section measurement exists in this region. E665 can check NMC measurements and extend the $\mathrm{Q}^{2}$ range for $0.006<\mathrm{X}_{\mathrm{bj}}<0.08$. The issues to be addressed in the measurement are:

trigger acceptance: under way, seem tractable

tracking: efficiency $90 \%$, tractable to $1 \%$

muon identification: effect of muon showers/old halo to be studied

vertex: tractable

spectrometer calibration: better than $0.5 \%$ (effect on cross section less than $2 \%$ )

electromagnetic: extension of work on $n / p$ ratio

backgrounds

radiative corrections: standard models have to include calorimeter bremsstrahlung veto normalization: under control ( using procedure of E98 ) 


\section{Budget Needs for $1993 / 94$}

The budget presented is less than optimal for the above tasks and fulfilling all Harvard's responsibilities. The reduction is made after discussion with other colleagues in the HE group. We had hoped during 1993 to continue our full involvement with jet reconstruction. As noted last year, we had wanted to hire a finishing graduate student as a Post doc to carry out the 'Altarelli' analysis on the 1990 and 1991 data. This was inhibited by budgetary constraints. If this is done by a graduate student from another university we will be involved in an advisory role (technology transfer).

The precise work on vector mesons depends critically on the vertex detector in the 1990 and 1991 data. This exciting work is just beginning. Ideally, therefore, we would continue budget for a post doc through the end of CY1994 to enable full realization of this work, which is a culmination of plans first made in proposals to Fermilab in 1969.

The absolute cross section, also a culmination of plans of 1969, will require the full time of Mr. Kotwal through CY1994, and hopefully a summer undergraduate assistant if the budget permits.

E665 computing is decentralized and each group has to provide computing capacity. The 1990 and 1991 data is 20 times the size of the 1987 data set. The Harvard group uses a Silicon Graphics computer (purchased in 1991) which is already saturated with one of the above analysis projects. If budget permits we would buy another SGI INDIGO computer with 65 MIPS for \$10K.

The group is now located permanently at Fermilab, because its size is too small to duplicate all common facilities at Harvard. However travel funds to present the exciting results will be increased over prior years. 


\section{The L3 Experiment}

(Professor Karl Strauch; Drs. Krishna Kumar, Andrey Kunin and Igor Vorobiev; Ian Scott)

The annihilation of high energy electrons and positrons has proven to be one of the most powerful probes into the nature of fundamental properties of matter. When CERN decided to build LEP, our group joined in 1980 in the planning of a LEP experiment with MIT colleagues (Professor Ting's group) with whom we had previously collaborated on the ISR experiment I209. Out of these discussions evolved the "Magnetic Hall" concept of the L3 experiment which was formally approved as one of four LEP experiments in September 1982. By the end of 1990, the collaboration included groups from Bulgaria, China, France, Germany, Hungary, India, Italy, The Netherlands, Spain, Sweden, Switzerland, Taiwan, USA, USSR. The U.S. participants came from Alabama, CIT, Carnegie-Mellon, Hurvard, Johns Hopkins, Lawrence Livermore, Los Alamos, MIT, Michigan, Northeastern, Oak Ridge, Princeton, Purdue, UC. San Diego.

The L3 detector is a general purpose detector with emphasis on accurate identification and energy measurement of photons, electrons and muons. It is installed at interaction point $\# 2$ on the LEP e+e- storage ring. LEP began operation in the Summer of 1989; both peak and average luminosities have increased with time. In 1992 peak luminosities of the nominal design value of $\mathrm{L}=10^{31} \mathrm{~cm}^{-2} \mathrm{sec}^{-1}$ were achieved. At this luminosity approximately one $\mathrm{Z}^{\mathrm{O}}$ is recorded every 4 seconds for a center-of-mass energy $\sqrt{ } s$ at the $Z^{\circ}$ peak. (1) its phase I LEP operates at a maximun center-of-mass energy $\sqrt{s}_{s}=100 \mathrm{GeV}$. In its phase II(1995-96), the maximum value of $\sqrt{ }_{s}$ will be increased to $\sim 190 \mathrm{GeV}$, above the $\mathrm{W}$ pair production threshold.

During our previous collaboration with the MIT group, we jointly developed a new type of drift chambers to measure the momentum of high energy muons. For reasons of effectiveness and efficiency, we again worked with our MIT colleagues (and others) on developing the precision muon detection system of L3. Harvard continues to do machine shop work for the forward, backward muon detector.

Our group is the major contributor to the study of the polarization of $\tau$ leptons produced in $Z^{\circ}$ decays. The value of the polarization of $\tau$ leptons in $e^{+} e^{-} \rightarrow \tau^{+} \tau^{-}(\gamma)$ is a sensitive probe of electroweak couplings. In particular, its measurement allows the determination of the ratio of the vector and axial-vector components of the couplings of the $\tau$ lepton to the weak neutral current. It provides an unambiguous test of parity violation in weak neutral current processes, and is a sensitive measure of the electroweak parameter $\sin ^{2} \theta_{w}$. 
The helicity of the $\tau$ leptons is analyzed by a study of the decay kinematics in the decay modes $\tau \rightarrow e v_{e} v_{\tau}, \mu v_{\mu} v_{\tau}, \pi(K) v_{\tau}, \rho v_{\tau}$ and $a_{1} v_{\tau}$ assuming a pure $(V-A)$ interaction for the $\tau$ charged current decay. A weighted average polarization of $-0.132 \pm 0.026$ (stat) \pm 0.21 (syst) has been measured. This corresponds to a ratio of the vector to the axial-vector coupling constant of the $\tau$ lepton to the weak neutral current of $\mathrm{gv} / \mathrm{g}_{\mathrm{a}}=0.068 \pm 0.019$. In the Standard Model, this leads to a value of $\sin ^{2} \theta_{w}=0.2326 \pm 0.0043$. These results are published in Physics Letters B294(1992); they were obtained with $400000 \mathrm{Z}^{\circ}$ decays collected in 1989. 91. This number has doubled with the events taken in 1992.

The results are expected to improve from the use of events in the endcap region. Furthermore, the measurement of the forward-backward polarization asymmetry will provide a sensitive test of lepton universality, and will effectively double the statistical precision of measurement of $\sin ^{2} \theta_{w}$. The Harvard group continues to play a leading role in the $\tau$ polarization analysis.

Mr. Scott started his work with our group by calibrating the muon spectrometer system. He has just completed his thesis on the $\tau$ polarization measurement using the data available up to the end of 1991. Drs. Kunin started as co-leader of the Higgs search group. He is concentrating more and more on the $\tau$ polarization measurement, and with $\mathrm{Dr}$. Vorobiev is very significantly improving particle identification and the accuracy of energy measurements, particularly in the BGO calorimeter. In January 1993 Dr. Kunin joined the MIT L3 group as Senior Research Associate. Dr. Vorobiev (from the Moscow ITEF L3 group) formally joined us at that time. Dr. Kumar joined us in 1990 and has greatly contributed to our successful concentration on the $\tau$ polarization measurement. Professor Strauch will retire from teaching at Harvard in 1993. He will continue to actively participate in L3 in future years. The Harvard L3 group will be reduced to himself after June 1994 (or even earlier) unless necessary support above that needed for the new members of the Harvard faculty becomes available. 


\section{Theory}

(Professor Roy Glauber)

The program of phenomenological analysis of high energy $\bar{p}$ cross sections begun in collaboration with members of the old UA4 group at CERN continues. The fits that have been found to the differential cross sections measured at the SPS--Collider suggest that further experimental attention must be paid to the small--momentum--transfer region of the differential cross section and to the the extrapolations made to its forward values.

Our analysis has in fact spurred a remeasurement of the small--momentum- -transfer cross sections that has been carried out in the Fall of 1991. The new collaboration UA4/2 will evidently succeed in reaching a considerably more accurate value of the ratio of the real and imaginary parts of the forward scattering amplitude. The value that seems to be emerging from this analysis is quite consistent with our models for the collision process, while the value reported earlier was not. The models we have been using should also be of value in dealing with the momentum transfer distributions for the process of diffractive dissociation.

In many high--energy collision experiments in which pions and kaons are multiply produced, it has been found that there is a distinct tendency for the particles of like charge to emerge with closely correlated momenta. This effect, which was predicted and searched for on the basis of a quantum optical analogy, has sometimes been called the "Bose-Einstein Effect", but more recently by the name of its optical analogue, the Hanbury-Brown-Twiss effect. That effect is a photon--photon correlation that is due, as we have shown many years ago, to the highly random (and incoherent) nature of the field excitations from ordinary light sources. (It tends to be completely absent in coherent sources.) The analysis of pion relative momentum distributions that has been carried out by analogy with the Hanbury-Brown-Twiss analysis makes implicit assumptions of incoherence that are likely to prove quite extreme in the elementary particle context --- for example, the assumption that the coherence length of the field excitations is infinitesimal compared to the dimensions of the collision volume. We propose to test these assumptions by means of simple field theoretical models, in order to see what the correlation effects may be telling us in more realistic terms about the collision processes.

Recently developed techniques for trapping ions and charged particles in time--varying electromagnetic fields are making new classes of experimental measuremerts possible, at unprecedentedly high levels of accurac $\%$. While the inspiration for the trapping schemes has usually come from classical calculation $x$, their mechanisms may be significantly influenced by 
quantum fluctuation effects. It will be necessary to understand the fluctuation effects in general, in order to deal with the cooling of the trapped particles, and for the precise analysis of experiments performed on them. We have been able to develop for this purpose a complete theory of the quantum mechanical behavior of particles trapped in an arbitrarily time-- varying quadrupole field. The analysis shows that for suitable ranges of the parameters the trapping is absolute; i.e., there is no quantum mechanical leakage. The non-stationary wave functions form a discrete sequence in one--to--one correspondence with the stationary states of a static harmonic oscillator. Coherent states can also be formed from the pseudostationary trapped states, and externally applied fields lead simply to transitions between these coherent states.

We are investigating the interactions of the trapped ions with the quantized electromagnetic field and have found that they are characterized in part by a paradoxical form of spontaneous emission process in which the trapped particle increases in excitation while radiating photons. This process indicates that radiative interactions tend to limit the lifetimes of trapped states; their stability is no longer absolute when radiation is taken into account. The lifetimes for states trapped by RF fields, however, can be quite long enough for their use in experiments. We have developed a new approximation method, akin to the Fermi pseudopotential approximation, for the treatment of light scattering by such time-dependent systems.

Several years ago we developed (in collaboration with P.Osland and M.Bleszynski) an asymptotic form of diffractive collision theory that applies to particle and nuclear collisions in the high energy limit. We have performed many calculations applying the scheme in various contexts and are preparing a lengthy review of the work. 


\section{Appendices: Bibliography and Budget Tables}

Immediately following this page are lists for each group of publications, conference talks, and doctoral theses. These lists cover the period from 1991 to the present.

Following the bibliographic tables are budget tables for our program. They include:

- Grant Application Budget Period Summary for 11/1/93-10/31/94

- Budget period summary broken down by group

- Budget summaries for remaining grant years (1993-1996)

- Equipment fund request detail

- Personnel list (showing fraction paid by DoE grant- 100\% if blank)

It should be noted that the $42 \%$ indirect cost rate is a special rate for the HEP program which has been negotiated with the Dean. It reflects the special needs of our program and is below the normal University rate of $69.5 \%$. 


\section{CDF Group \\ Publications}

1. "Measurement of the Average Lifetime of B-hadrons Produced in ppbar Collisions at $\sqrt{s}=1.8 \mathrm{TeV}$," F. Abe et al., The CDF Collaboration, to be submitted to Phys. Rev. Lett. (1993).

2. "Observation of the Decay $B_{a}^{0} \rightarrow J / \psi \phi$ in $\bar{p} p$ Collisions at $\sqrt{s}=1.8 \mathrm{TeV}$," F. Abe et al., The CDF Collaboration, to be submitted to Phys. Rev. Lett. (1993)

3. "Search for Quark Compositeness, Axigluons and Heavy Particles using the Dijet Invariant Mass Spectrum Observed in $\bar{p} p$ Collisions," F. Abe et al., The CDF Collaboration, to be submitted to Phys. Rev. Lett. (1993).

4. "A Measurement of the B Meson and b Quark Cross Sections at $\sqrt{s}=1.8 \mathrm{TeV}$ Using the Exclusive Decay $B^{0} \rightarrow J / \psi K^{*}(882)^{0}$," F. Abe et al., The CDF Collaboration, submitted to Phys. Rev. D. (1993).

5. "A Measurement of the Bottom Quark Production Cross Section in $1.8 \mathrm{TeV}$ ProtonAntiproton Collisions Using Semileptonic Decays to Muons," F. Abe et al., The CDF Collaboration, to be submitted to Phys. Rev. Lett. (1993).

6. "Inclusive $\chi_{c}$ and b-quark Production in $\bar{p} p$ Collisions at $\sqrt{s}=1.8 \mathrm{TeV}, " F$. Abe et al., The CDF Collaboration, submitted to Phys. Rev. Lett. May 20, 1993. FERMILABPUB-93/106-E.

7. "A Search for First-Generation Leptoquarks in $\bar{p} p$ Collisions at $\sqrt{s}=1.8 \mathrm{TeV}$ at CDF," F. Abe et al., The CDF Collaboration, submitted to Phys. Rev. D, Rapid Communications, April 1, 1993; FERMILAB-PUB-93/070-E.

8. "A Measurement of Jet Multiplicity in W Events Produced in $\bar{p} p$ Collisions at $\sqrt{8}=1.8$ TeV," F. Abe et al., The CDF Collaboration, submitted to Phys. Rev. Lett. March 25, 1993.

9. "The Center-of-Mass Angular Distribution from Prompt Photons Produced in $\bar{p} p$ Collisions at $\sqrt{s}=1.8 \mathrm{TeV}, " \mathrm{~F}$. Abe et al., The CDF Collaboration, submitted to Phys. Rev. Lett. March 1, 1993. FERMILAB-PUB-93/-32-E.

10. "A Study of Four-Jet Events and Evidence for Double Parton Interactions in $\bar{p} p$ Collisions at $\sqrt{s}=1.8 \mathrm{TeV}, " \mathrm{~F}$. Abe et al., The CDF Collaboration, submitted to Phys. Rev. D, January 8, 1993. (accepted March 22nd) FERMILAB-PUB-93/003-E.

11. "Measurement of the Cross Section for Production of Two Isolated Prompt Photons in $\bar{p} p$ Collisions at $\sqrt{\delta}=1.8 \mathrm{TeV}, "$ Phys. Rev. Lett. 70, 2232 (1993).

12. "A Prompt Photon Cross Section Measurement in $\bar{p} p$ Collisions at $\sqrt{s}=1.8 \mathrm{TeV}, " F$. Abe et al., The CDF Collaboration, to be submitted to Phys. Rev. D. 
13. "Measurement of the Dijet Mass Distribution in pp Collisions at $\sqrt{8}=1.8 \mathrm{TeV}, " \mathrm{~F}$. Abe et al., The CDF Collaboration, submitted to Phys. Rev. D February 16, 1993.

14. "Search for $\Lambda_{b}->J / \psi \Lambda^{0}$ in pp Collisions at $\sqrt{\delta}=1.8 \mathrm{TeV}, " F$. Abe et al., The CDF Collaboration, Physical Review D 47, Rapid Communications 47, R2639 (1993).

15. "Comparion of Jet Production in $\bar{p}$ Collisions at $\sqrt{8}=546$ and $1800 \mathrm{GeV}, " \mathrm{~F}$. A be et al., Phys. Rev. Lett. 70 (1993).

16. "Analysis of Jet Charged Momentum Distributions for Quark-Gluon Separation in pp Collisions at $\sqrt{8}=1.8 \mathrm{TeV}, " \mathrm{~F}$. Abe et al., The CDF Collaboration, to be submitted to Phys. Rev, D.

17. "Inclusive $J / \psi, \psi^{\prime}$ and b-Quark Production in $\bar{p} p$ Collisions at $\sqrt{s}=1.8 \mathrm{TeV}, " \mathrm{~F}$. A be et al., The CDF Collaboration, Phys. Rev. Lett. 69, 3704 (1982).

18. "Search for Squarks and Gluinos from $\bar{p}$ Collisions at $\sqrt{\delta}=1.8 \mathrm{TeV}, " \mathrm{~F}$. Abe et al., The CDF Collaboration, Phys. Rev. Lett. 69, 3439 (1992).

19. "Measurement of Drell-Yan Production in $\bar{p} p$ Collisions at $\sqrt{8}=1.8 \mathrm{TeV}, " \mathrm{~F}$. Abe et al., The CDF Collaboration, to be submitted to Phys. Rev. Lett.

20. "Limits on the Production of Mastive Stable Charged Particles," F. Abe et al., The CDF Collaboration, Phys. Rev. D, Rapid Communications, 46, R1889 (1982).

21. "A Measurement of Jet Shepes in $p p$ Collisions at $\sqrt{8}=1.8$ Tev," F. Abe et al., The CDF Collaboration, Phys. Rev. Lett. 70, 713 (1993).

22. "Limit on the Rare Decay $W^{ \pm} \rightarrow \gamma+\pi^{ \pm}$in $\overline{p p}$ Collisions at $\sqrt{s}=1.8 \mathrm{TeV}, " \mathrm{~F}$. Abe et al., The CDF Collaboration, Phys. Rev. Lett. 69, 2160 (1982).

23. "A Measurement of the Bottom Quark Production Cross Section Using Semileptonic Decay Electrons in pp Collisions at $\sqrt{\delta}=1.8 \mathrm{TeV}, " \mathrm{~F}$. Abe et al., The CDF Collaboration, submitted to Phys. Rev. Lett. April 10, 1983. FEMRILAB-PUB-93/091-E.

24. "The Dijet Angular Distribution at $\sqrt{s}=1.8 \mathrm{TeV}, " \mathrm{~F}$. Abe et al., The CDF Collaboration, Phys. Rev. Lett. 69, 2897 (1982).

25. "A Measurement of the Production and Muouic Decay Rate of $\mathrm{W}$ and $\mathrm{Z}$ Bosons in $\bar{p} p$ Collisions at $\sqrt{8}=1.8 \mathrm{TeV},{ }^{n} \mathrm{~F}$. Abe et al., The CDF Collaboration, Phys. Rev. Lett. 69,28 (1992).

26. "Measurement of the Isolated Prompt Photon Cross Section Measurement in $\bar{p} p$ Collisions at $\sqrt{s}=1.8 \mathrm{TeV}, " \mathrm{~F}$. A be et al., The CDF Collaboration, Phys. Rev. Lett. 68, 2734 (1992).

27. "A Measurement of the B Meson and b Quark Cross Section at $\sqrt{8}=1.8$ TeV Using the Exclusive Decay $B^{ \pm} \rightarrow J / \psi K^{ \pm}, " F$. Abe et al., The CDF Collaboration, Phys. Rev. Lett. 68, 3403 (1982). 
28. "A Limit on the Top Quark Mass from Proton-Antiproton Collisions at $\sqrt{8}=1800$ GeV," F. Abe et al., the CDF Collaboration, Phys. Rev. D. 45, 3921 (1992).

29. "A Search for New Gauge Bosons in pop Collisions at $\sqrt{\delta}=1.8 \mathrm{TeV}, "$ F. A be et al., the CDF Collaboration, Phys. Rev. Lett. 68, 1463 (1982).

30. "Properties of Events with Large Total Transverse Energy Produced in ProtonAntiproton Collisions at $\sqrt{s}=1.8 \mathrm{TeV}, " \mathrm{~F}$. Abe et al., The CDF Collaboration, Phys. Rev. D. 45, 2249 (1982).

31. "A Lower Limit on the Top Quark Mass from Events with Two Leptons in p pbar Collisions at $\sqrt{8}=1.8 \mathrm{TeV}, " \mathrm{~F}$. Abe et al., The CDF Collaboration, Phys. Rev. Lett. 68, 447 (1982).

32. "Measurement of the Ratio $\sigma \bullet B(W \rightarrow \tau \nu) / \sigma \bullet B(W \rightarrow e \nu)$ in $p p$ Collisions at $\sqrt{s}=$ $1.8 \mathrm{TeV}$, as a Test of Lepton Universality," F. A be et al., The CDF Collaboration, Phys. Rev. Lett. 68, 3398 (1992).

33. "Menurement of $B^{\circ} B^{\circ}$ Mixing at the Fermilab Tevatron Collider," F. A be et a., The CDF Collaboration, Phys. Rev. Lett. 67, 3351 (1991).

34. "Search for $W^{\prime} \rightarrow e \nu$ and $W^{\prime} \rightarrow \mu \nu$ in pp Collinions at $\sqrt{s}=1.8 \mathrm{TeV}, "$ F. Abe et al., The CDF Collaboration, Phys. Rev. Lett. 67, 2609 (1891).

35. "Inclusive Jet Cross Section in $\mathrm{pp}$ Collisions at $\sqrt{8}=1.8 \mathrm{TeV}$, " F. Abe et al., The CDF Collaboration, Phys. Rev. Lett. 68, 1104 (1982).

36. "Topology of Three Jet Events in pp Collisions at $\sqrt{8}=1.8 \mathrm{ToV}, "$ F. A be et al., The CDF Collaboration, Phys. Rev. D 45, 1448 (1982).

37. "Lepton Asymmetry in W Decays from pp Collisions at $\sqrt{8}=1.8 \mathrm{Tev}, "$ F. Abe et al., the CDF Collaboration, Phys. Rev. Lett. 68, 1458 (1982).

38. "Measurement of the $e^{+} e^{-}$Invariant Mass Distribution in $\bar{p} p$ Collisions at $\sqrt{8}=1.8$ TeV," F. Abe et al., the CDF Collaboration, Phys. Rev. Lett. 67, 2418 (1991). PUB-91/169-E.

39. "Measurement of the $Z$ pT Distribution in $\overline{p p}$ Collisions at $\sqrt{s}=1.8 \mathrm{TeV}, " \mathrm{~F}$. Abe et al., The CDF Collaboration, Phys. Rev. Lett. 67, 2837 (1891).

40. "Measurement of the W Boson $P_{T}$ Distribution in $\bar{p}$ Colliaions at sqrt $(\mathrm{s})=1.8 \mathrm{TeV}$, F. A be et al., Phys. Rev. Lett. 66, 2951 (1891).

41. "A Measurement of $\sigma(W \rightarrow e \nu)$ and $\sigma\left(Z^{0} \rightarrow e^{+} e^{-}\right)$in $\overline{p p}$ Collisions at $\sqrt{s}=1800$ GeV," F. Abe et al., Phys. Rev. D 44, 29 (1981).

42. "A Determination of $\sin ^{2} \theta_{W}$ from the Forward-Backward Asymmetry in $\overline{p p} \rightarrow Z^{0} X \rightarrow$ $e^{+} e^{-X}$ Interactions at $\sqrt{8}=1.8 \mathrm{TeV},{ }^{n}$ F. Abe et al., Phys. Rev. Lett. 67, $1502(1981)$. 
43. "Measurement of QCD Jet Broadening in pp Collisions at $\sqrt{\mathrm{s}}=1.8 \mathrm{TeV}, " \mathrm{~F}$. A be et al., Phys. Rev. D 44, 601 (1991).

44. "Top Quark Search in the Electron + Jets Channel in Proton-Antiproton Collisions at $\sqrt{\delta}=1.8 \mathrm{TeV}$," F. Abe et al., Phys. Rev. D. 43, 664 (1991).

45. "A Measurement of the W Boson Mass in $1.8 \mathrm{TeV} \bar{p} p$ Collisions," F. Abe et al., Phys. Rev. D. 43, 2070 (1981).

46. "Toward a Standarization of Jet Definitions," by John E. Huth et al., FERMILABCONF-80-249-E, Dec 1990. 6pp. Presented at Summer Study on High Energy Physics, Reaearch Directions for the Decade, Snowmass, CO, Jun 25 - Jul 13, 1990.

\section{Conference Talke}

1. "QCD Tests at CDF," The CDF Collaboration, talk given by J. Huth, U.S. Department of Energy, Germantown, MD, January 26, 1993.

2. "Tau Physics at pp Colliders," The CDF Collaboration, J. Konigsberg, published Proceedings 2nd Workshop on Tau Lepton Phyuics, The Ohio State University, Columbus, OH, September 8-11, 1982. FERMILAB-CONF-83/008-E.

3. "CDF Electroweak Results," The CDF Collaboration, M. Franklin, published Proceed. ings XXVIth International Conference on High Energy Physics, Dallas, TX, August 6-12, 1992.

4. "CDF Top Search," The CDF Collaboration, J. Huth, published Proceedinge XXVIth International Conference on High Energy Physics, Dalla, TX, August 6-12, 1992.

5. "CDF Top Search," talk given by J. Huth, XXVIth International Conference on High Energy Physics, Dallas, TX, August 6-12, 1892.

6. "Top and Precision Electroweak Physics with the Main Injector," talk given by J. Huth, HEPAP Subpanel Meeting, Fermilab, Batavia, IL, February 28, 1982.

7. "Status of the Fermilab Collider Program," John Huth, Presented at the 1991 Symponium on the SSC, Corpus Christi, Texa, Oct. 13-17 1991.

8. "Results from pp Colliders," J. Huth, published Proceedings Particles and Fields '91, Univeraity of Vancouver, Vancouver, British Columbia, Canada, August 18-22, 1991. FERMILAB-CONF-91/223-E.

9. "Precision Tests of the Standard Model at CDF," The CDF Collaboration, P. Schlabach, published Proc. Joint Intl. Lepton-Photon Symp. and Europhysice Conf. on High Energy Phyuics," Geneve, Switzerlend, July 25-August 1, 1981.

\section{Doctoral Thesie}

1. "Measurement of the $Z$ Boson Tranuverse Momentum Distribution at the Tevatron," J. Ng, Thesis Submitted to Harvard University, Cambridge, MA, May 1991. 


\section{CLEO Group}

Publications

1. Exclusive and Inclusive Semileptonic Decays of B Mesons to D Mesons

R. Fulton et al., Physical Review D 43, 651 (1991)

2. Study of $\pi^{+} \pi^{-}$Transitions from the $\Upsilon(3 S)$ and a Search for the $h_{b}$

I.C. Brock et al., Physical Review D 4s, 1448 (1991)

3. Study of $D^{0}$ Decays into Final States with a $\pi^{0}$ or $\eta$

K. Kinoshita et al., Physical Review D 48, 2836 (1991)

4. Incluaive Production of the Charmed Baryon $\Lambda_{c}^{+}$from $e^{+} e^{-}$Annihilations at $\sqrt{s}=$ $10.55 \mathrm{GeV}$

P. Avery et al., Phyaical Review D 48, 3509 (1891)

b. Study of Continuum $D^{*+}$ Spin Allignment

Y. Kubota et al., Physical Review D 44, 593 (1901)

6. Measurement of the Inclusive $B^{*}$ Cross Section above the $\Upsilon(4 S)$

D. S. Akerib et al., Phyoical Review Letters 67, 1002 (1891)

7. Inclusive $\chi(2 P)$ Production in $\Upsilon(3 S)$ Decay

R. Morrison et al., Physical Reviow Letters 67, 1696 (1891)

8. Unusual Decay Modes of $D^{0}$ and $D^{+}$Mesons

R. Ammar et al., Physical Review D 44, 3383 (1891)

9. Messurement of the Ratio $B\left(D^{0} \rightarrow K^{*-} e^{+} \nu_{c}\right) / B\left(D^{0} \rightarrow K^{-} e^{+} \nu_{0}\right)$

G. Crawford et al., Physical Review D 44, 3394 (1991)

10. Inclunive and Exclusive Decays of B Mesons to Final States Including Charm and Charmonium Mesons

D. Bortoletto et al., Physical Review D 48, 21 (1892)

11. Measurenient of Baryon Production in B-Meson Decay

G. Crawford et al., Phyoical Review D 46, 752 (1992)

12. $D_{0}^{+}$decays to $\eta \pi^{+}$and $\eta^{\prime} \pi^{+}$

P. Avery et al., Physical Review Letters 68, 1275 (1992)

13. $D_{\text {s }}^{+}$decaye to $\eta \rho^{+}, \eta^{\prime} \rho^{+}$, and $\phi \rho^{+}$

P. Avery et al., Physical Review Lettern 68, 1270 (1992)

14. Measurements of Semileptonic Branching Fractions of B Mesons at the $\Upsilon(4 S)$ Resonance

S. Henderson et al., Physical Reviow D 48, 2212 (1982)

15. Two-body $D_{0}^{+}$decays to $\eta \pi^{+}, \eta^{\prime} \pi^{+}, \eta \rho^{+}, \eta^{\prime} \rho^{+}$, and $\phi \rho^{+}$

M. Deoudi et al., Physical Review D 46, 3965 (1982) 
16. Electronic Branching Ratio of the $r$ lepton

R. Ammar et al., Physical Review D 46, 3976 (1992)

17. Observation of the Decay $\Xi_{c}^{0} \rightarrow \Omega^{-} K^{+}$

S. Henderson et al., Phyaics Letters B 283, 16 (1982)

18. The CLEO II detector

Y. Kubote et al, Nuclear Inotruments and Mothods A 320, 66 (1982)

19. A Menourement of the tau lepton lifetime

M. Battle et al., Phyaico Letters B 201, 488 (1992)

20. Measurement of the $D^{*}(2010)$ Brunching Fraction

F. Butler et al., Phyoical Reviow Letters 00, 2041 (1902)

21. Isospin Mass Splittings from Precinion Measurements of $D^{*}-D$ Mass Differences

D. Bortoletto et al., Phyaical Review Letters 60, 2046 (1892)

22. Shape Studies of Quark Jets vs. Gluon Jets at $\sqrt{\mathrm{a}}=10 \mathrm{GeV}$

M. S. Alem et al., Phyaical Reviow D 46, 4822 (1902)

23. Exclunive $\chi(2 P)$ Production in $\Upsilon(3 S)$ Decay

G. Crawford ot al, Phyuica Letteru B 204, 139 (1992)

24. Messurement of $r$ Decays Involving $\eta$ Mesons

M. Artuso et al., Phyuical Roview Lettero 00, 3278 (1892)

25. Mesurement of the Thu Lepton Electronic Branching Fraction

D. S. Akerib et al., Phyaical Review Letterı 60, 3610 (1902)

26. Search for $\tau^{-} \rightarrow \gamma^{-}$: A Test of Lepton Number Conservation

A. Bean et al., Phyaical Review Letters 70, 138 (1993)

27. Tuu Decays with One Charged Particle plus Multiple $\pi^{0} \cdot s$

M. Procario et al., Phyoical Review Letters 70, 1207 (1993)

28. Lepton As:'mmeiry Measurements in $B \rightarrow D^{*} / \nu_{1}$ and Implications for V.A and the Form Factore

S. Sanghera et al., Phyoical Review D 47, 791 (1993)

29. Study of $D^{0}$ decayo into $K^{0}$ and $K^{* 0}$

M. Procario et al., CLNS 92/1167, CLEO92-10

(submitted to Phyaical Review D)

30. Search for Excluaive $b \rightarrow u$ Semileptonic Decays of $B$ Mesone

A. Bean et al., CLNS 92/1174, CLEO 82-12

(submitted to Phyoical Review Letters ) 
31. Production and Decay of the $D_{11}(2536)^{+}$

J.P. Alexander et al, CLNS 93/1191, CLEO 93-1

(oubmitted to Physica Letters B)

32. A Menurement of the Tau Lepton Masu

R. Ballest et al., CLNS 93/1104, CLEO 93-2

(aubmitted to Phyuical Review Letters )

33. A Limit on the Tau Neutrino Mase

D. Cinabro et al., CLNS 93/1105, CLEO 03-3

(aubmitted to Physical Review Letters )

Conference Tulke

1. IR beampipe upgrade

Hitoshi Yamamoto, HEPAP review (CESR/CLEO), Feb. 1992

2. $B_{d}$ mixing by partial reconotruction tas Mike Saulnjer, APS Meeting, Weubington, April 1002

3. Searches for B $-i$ lepton + neutrino

Dave Cinabro, ICHEP, Dallen, Auguat 1092

4. CESR/CLEO interaction reglon upgrade

Stu Henderson, ICHEP, Dalle, Auguat 1092

8. Recent Results from CLEO

Hitoshi Yamamoto, San Miniato Conference, June 1992

6. CESR/CLEO IR upgrade

Hitoobs Yamamoto, APS/DPF Meeting, Formilab, Nov, 1992

7. Tau mese and Tau neutrino mess

Stu Henderson, APS/DPF Meeting, Fermilab, Nov, 1902

8. Silicon Vertex Detector for CLEO-II

Dave Cinabro, APS/DPF Meeting, Fermilab, Nov, 1992

9. CESR/OLEO IR upgrade

Hitonhi Yamamoto, KEK B-factory workehop, Nov, 1092

10. CLEO-II dE/dX menurement

Kay Kinoshita, KEK B.factory workahop, Nov, 1992

11. IR/beampipe upgrade

Hitouhi Yamemoto, PAC review (CESR/CLEO), Feb. 1993

12. Lifetime ratio of $B^{-}$and $B^{0}$ by partial reconatruction tag Mike Saulnjer, APS Meeting, Wahington, April 1983 
13. Measurement of Doubly-Cabbibo-supprensed decay of $D^{0}$

Te-hui Liu, APS Meeting, Washington, April 1893

14. Reviow of $B$ decays, measurements and predictions

Hitoshi Yamamoto, B-phyaicı at Hadron Acceleratora, Snowmans, June 1993

15. Charmed meson decays

Hitorhi Yamamoto, Heavy Flavor Conference, Montreal, July 1983

16. B meson decays

Kay Kinonhita, Heavy Flavor Conference, Montreal, July 1993

\section{Doctoral Theals}

1. Study of Lambda-like Baryons in $e^{+} e^{-}$Annihilation

J. Wollnoks, Harvard Univerdity, May 1001 


\section{Neutrino Group \\ Publicatione}

1. "Deep Inelaetic Scattering",

S. Michra et al. (A Review Volume in preparation in collaboration with G. Altarelli, G. Smadja, M. Virchaux, R. Voat, and D.von Harrach.), World Scientific Publication, to be completed by the end of this year.

2. "A Review of Recent Results from the CCFR Collaboration",

S. Mishra, A review article in preparation for the International Journal of Modern Phyaics, $A$, to be completed by the end of this year.

3. "Neutrino Tridents and W-Z Interference", S.R. Miohre et al., Phys.Rev.Lett., 68, 3117(1091).

4. "Nucleon Structure Functions from High Energy Noutrino Interactions with Iron uting Narrow Band Beam",

E. Oltman et al., Z. Phys.C, C68, B1(1992).

8. "Search for Right Handed Coupling in $\nu \cdot N$ Interactiona", S.R. Miohra et al., Phys.Rev.Lett., 08, 3480(1902).

6. "Comparicon of the Nucleon Structure Function, $F_{2}\left(m, Q^{2}\right)$, and the Mean Square Charge Test",

S.R.Miehre et al., Submitted for publication in Phyo.Rev.Lett.

7. "A Precinion measurement of the Gross-Llewellyn Smith Sum Rule", W.C.Leung et al., Submitted for publication in Phys.Rev.Lott.

8. "A Measurement of $\Lambda_{Q C D}$ from $\nu \cdot$ Fe Non-ninglet Structure Functions at the Fermilab Tevatron", P.Z.Quintas et al., Submitted for publication in Phys.Rev.Lett.

9. "A Meanurement of ToV Muon Energy Lose in Iron", W.K.Sakumoto et al., Phyı. Rev. D, 16, 3042(1992).

10. "Neutrino Production of Same Sign Dimuona", P.H. Sandler et al., Z. Phya. C87, Vol.\#1, 1(1093).

11. "Measurement of the Strange Sea Distribution Uaing Neutrino Charm Production ", S.A. Rabinowits et al., Phys. Rev. Lett. 70, \#2, 134(1993).

12. "Measuring Muon Momente with the CCFR Neutrino Detector", B.J.King et al., Nucl. Inot. Meth. As02, 284(1901).

13. "Deaign and Implementation of Aigh Precinion Readout Syatem for the Zeus Calorimeter",

A.Culdwell ot al., Novis Preprint \# 1442, Fob(1901); Submitted for publication in Nucl. Inut. Meth. 


\section{Conference Talk»}

1. "Recent Electroweak Results by the CCFR Collaboration", presented at Moriond Electroweak '91 Conference, Les Arc, France, March-1991.

2. "Preciaion Measurements of $\nu$. Fe Structure functiona, $F-2\left(x, Q^{2}\right)$ and $x F_{3}\left(x, Q^{2}\right)$, and Teats of QCD at the Fermilab Tevatron", presented at the Lepton-Photon '91 Conference, CERN, Geneva, Jul-1991.

3. "Preciaion Tests of the Electroweak Unification in $\nu$-Nucleon Scattering at the Fermilab Tevatron",

presented at the Lepton-Photon '91 Conference, CERN, Geneva, Jul-1991.

4. "Preciaion Meanurements of $v$-Fe Structure functione, $F-2\left(x, Q^{2}\right)$ and $x F_{3}\left(x, Q^{2}\right)$, and Teats of QCD at the Fermilab Tevatron", presented at the SLAC Topical Conference '91, SLAC, Stanford, USA, AUg-1991.

8. "Precinion Measurements of $\nu$. Fe Structure functiona, $F-2\left(x, Q^{2}\right)$ and $\infty F_{3}\left(x, Q^{2}\right)$, and Touts of QCD at the Fermilab Tovatron", presented at the Division of Particle and Fieldo (DPF) '91 Conference, Vancouver, Canada, Aug-1891.

6. "Precinion Teats of the Electroweak Unification in $\nu$-Nucleon Scattering at the Fermilab Tovatron", presented at the Division of Particle and Fieldo (DPF) '91 Conference, Vancouver, Cureda, Aug-1091.

7. "Nucleon Structure Functions and Tests of QCD in High Energy Neutrino Scattering", presented at the XXVI International Conference on High Energy Physics, Dalles, 1992.

8. "Precicon Measurement of $\sin ^{2} \theta_{w}$ in Neutrino Scattering", presented at the XXVI International Conference on High Energy Physics, Dallas, 1982.

9. "Neutrino Production of Dimuone", presented at the XXVI International Conference on High Energy Physica, Dallas, 1892.

10. "A Review of Precioion Mesurements of $\sin ^{2} \theta w$ in $\nu$-N Scattering", S.R.Minhra; invited review talk to be presented at the International Conference on Neutral Currents Tewenty Yours Later, Paria, July 1993.

11. "Nucleon Structure Functions from Neutrino-Nucleon Scattering", S.R.Mishra; invited review talk to be presented at the Neutrinos in the Nuclear, AstroParticle Physics and Cosmology, Erice, Sep 1983. 


\section{E665 Group \\ Publications}

1. M.R. Adams et al., Distribution of Charged Hadrons Observed in Deep-Inelastic Muon-Deuterium Scattering at $490 \mathrm{GeV}$, Phys. Lett B272, 163 (1991).

2. M.R. Adam et al., Saturation of Shadowing at Very Low $x_{b j}$, Phys. Rev. Lett. 68, 3266 (1992).

3. M.R. Adams et al., Firot Measurement of Jet Production Rates in Deep-inelastic Lepton-Proton Scattering, Phys. Rev. Lett. 69, 1026 (1992).

4. M.R. Adams et al., Shadowing in the muon-xenon inelastic scattering crous section at $490 \mathrm{GeV}$, Phys. Lett. B287, 375 (1992).

5. M.R. Adams et al., An Investigation of Bose-Einstein Correlations in Muon-Nucleon Interactions at $480 \mathrm{GeV}$, accepted for publication in Phys. Lett. B (1893).

6. M.R. Adams et al., Mesourement of the ratio $\sigma_{n} / \sigma_{p}$ in inelastic muon-nucleon scattering at very low and $Q^{2}$, submitted to Phys. Lett. B (1993).

7. M.R. Adams et al., Perturbative QCD Effects Observed in $490 \mathrm{GeV}$ Deep-Inelastic Muon Scattering.

8. M.R. Adams et al., $Q^{2}$ Dependence of the Average Squared Tranoverse Energy of Jets in Deep-Inelestic Muon-Nucleon Scattering with Comparion to QCD Predictions.

9. M.R. Adams et al., Scaled Energy (8) Distributions of Charged Hadrons Observed in Deep-Irelantic Muon Scattering at $490 \mathrm{GeV}$ from Xenon and Deuterium Targets.

Conference Talks

1. M. Schmitt et al., Muon Interactions at $490 \mathrm{GeV}$, FERMILAB-Conf-90/126-E, Proceedings of the XXVth Rencontre de Moriond, ed. by J. Tran Thanh Van, (Edition Frontieres, Gif-sur Yvette Cedex) p. 375 (1991).

2. J. Conrad et al., Di-jet Production in the Forward CMS From Deep Inelaetic Muon Scattering, Proceedings of The XXVII Rencontres de Moriond, QCD and High Energy Hadronic Interactions, March 1892.

3. J.M. Conrad et al., Jet Production in Deep Inelatic Muon Scattering, Meeting of the American Phyuical Society Division of Particles and Fields, Batavia, IL, Nov, 1992

\section{Doctoral Theses}

1. Michael H. Schmitt, Deep Inelestic Exclusive po Production Using 485 GeV Muons, Doctoral Thesis - Harvard University (1991).

2. Janet Conrad, A Study of the $Q^{2}$ Dependence of the QCD Coupling Conatant from the Tranoverse Momentum of Jets in Deep Inelastic Muon Scattering, Doctoral Thesis - Harvard University (1903). 


\section{L3 Group \\ Publications}

1. L3 Collab., B. Adeva et al., Phys. Lett. B 257 (1991) 469.

Determination of $\alpha_{\mathrm{B}}$ from Energy-Energy Correlations Measured on the $\mathrm{Z}^{0}$ Resonance

2. L3 Collab., B. Adeva et al., Phys. Lett. B 257 (1991) 450.

Search for the Neutral Higgs Boson

3. L3 Collab., B. Adeva et al., Phys. Lett. B 259 (1991) 199.

Measurement of the Inclusive Production of Neutral Pions and Charged Particles on the $\mathrm{Z}^{0}$ Resonance

4. L3 Collab., B. Adeva et al., Phys. Lett. B 261 (1991) 169.

Search for Leptoquarks in $\mathrm{Z}^{0}$ Decays

5. L3 Collab., B. Adeva et al., Phys. Lett. B 261 (1991) 177.

Measurement of $Z^{0} \rightarrow b \bar{b}$ Decays and the Semileptonic Branching Ratio $\operatorname{Br}(b \rightarrow l+X)$

6. L3 Collab., B. Adeva et al., Z. Phys. C 51 (1991) 179.

Measurement of Electroweak Parameters from Hadronic and Leptonic Decays of the $\mathrm{Z}^{0}$

7. L3 Collab., B. Adeva et al., Phys. Lett. B 262 (1991) 155

Search for Narrow High-Mass Resonances in Radiative Decays of the $\mathrm{Z}^{0}$

8. L3 Collab., B. Adeva et al., Phys. Lett. B 263 (1991) 551

A Test of QCD based on 3-Jet Events from $Z^{0}$ Decays

9. L3 Collab., B. Adeva et al., Phys. Lett. B 265 (1991) 451.

Decay Properties of Tau Leptons Measured at the $Z^{0}$ Resonance

10. L3 Collab., B. Adeva et al., Phys. Lett. B 270 (1991) 111.

Measurement of the Lifetime of B-Hadrons and a Determination of $\left|V_{c b}\right|$

11. L3 Collab., B. Adeva et al., Phys. Lett. B 271 (1991) 461.

Measurement of the Strong Coupling Constant $\alpha_{b}$ for Bottom Quarks at the $Z^{0}$ Resonance

12. L3 Collab., B. Adeva et al., Phys. Lett. B 271 (1991) 453.

Search for Lepton Flavour Violation in $\mathrm{Z}^{0}$ Decays

13. L3 Collab., B. Adeva et al., Phys. Lett. B 275 (1992) 209.

A Direct Determination of the Number of Light Neutrino Families from $e^{+} e^{-} \rightarrow \nu \bar{\nu} \gamma$ at LEP

14. The LEP Collaborations: ALEPH, DELPHI, L3 and OPAL, Phys. Lett. B 276 (1992) 247.

Electroweak Parameters of the Z Resonance and the Standard Model 
15. L3 Collab., B. Adeva et al., Phys. Lett. B 283 (1992) 454.

Search for the Neutral Higgs Boson at LEP

16. L3 Collab., B. Adeva et al., Z. Phys. C 55 (1992) 39.

Studies of Hadronic Event Structure and Comparisons with QCD Models at the $\mathrm{Z}^{0}$ Resonance

17. L3 Collab., O. Adriani et al., Phys. Lett. B 284 (1992) 471.

Determination of $\alpha_{\boldsymbol{s}}$ from Hadronic Event Shapes Measured on the $\mathrm{Z}^{0}$ Resonance

18. L3 Collab., O. Adriani et al., Phys. Lett. B 286 (1992) 403.

Measurement of Inclusive $\eta$ Production in Hadronic Decays of the $\mathrm{Z}^{\mathbf{0}}$

41 L3 Collab., B. Adeva et al., Phys. Lett. B 288 (1992) 395. An Improved Measurement of $B^{0}-\bar{B}^{0}$ Mixing in $Z^{0}$ Decays

19. L3 Collab., O. Adriani et al., Phys. Lett. B 288 (1992) 404. A Test of Quantum Electrodynamics in the Reaction $e^{+} e^{-} \rightarrow \gamma \gamma(\gamma)$

20. L3 Collab., O. Adriani et al., Phys. Lett. B 288 (1992) 412. Inclusive $\mathrm{J}$ production in $\mathrm{Z}^{0}$ Decays

21. L3 Colląb., O. Adriani et al., Phys. Lett. B 292 (1992) 454. Measurement of the $e^{+} e^{-} \rightarrow b \bar{b}$ and $e^{+} e^{-} \rightarrow c \bar{c}$ Forward-Backward Asymmetries at the $\mathrm{Z}^{0}$ Resonance

22. L3 Collab., O. Adriani et al., Phys. Lett. B 292 (1992) 463.

Determination of the number of light neutrino species

23. L3 Collab., O. Adriani et al., Phys. Lett. B 292 (1992) 472.

Isolated Hard Photon Emission in Hadronic $\mathrm{Z}^{0}$ Decays

24. L3 Collab., O. Adriani et al., Phys. Lett. B 294 (1992) 466.

A Measurement of $\tau$ Polarization in $\mathrm{Z}^{0}$ Decays

25. L3 Collab., O. Adriani et al., Phys. Lett. B 294 (1992) 457. Searches for Non-Minimal Higgs Bosons in $\mathrm{Z}^{0}$ Decays

26. L3 Collab., O. Adriani et al., Phys. Lett. B 295 (1992) 337. High Mass Photon Pairs in $\ell^{+} \ell^{-} \gamma \gamma$ events at LEP

27. L3 Collab., O. Adriani et al., Z. Phys. C 57 (1993) 355. Search for Non-Minimal Higgs Bosons in $\mathrm{Z}^{0}$ Decays

28. L3 Collab., O. Adriani et al., Phy " "ett. B 295 (1992) 371. Search for Isosinglet Neutral Hea eptons in $\mathrm{Z}^{0}$ Decays

29. L3 Collab., O. Adriani et al., Phys. Lett. B 297 (1992) 469.

Search for Anomalous Production of Single-Photon Events in $e^{+} e^{-}$-Annihilations at the $\mathrm{Z}$ Resonance 
30. L3 Collab., O. Adriani et al., Phys. Lett. B 301 (1993) 136.

Determination of Quark Electroweak Couplings from Direct Photon Production in Hadronic Z Decays

31. L3 Collab., O. Adriani et al., Phys. Lett. B 303 (1993) 391.

A Search for the Neutral Higgs Boson at LEP

32. L3 Collab., O. Adriani et al., Phys. Rep. ??? (1993) ???

CERN preprint CERN-PPE/93-31; February 22, 1993.

Results from the L3 Experiment at LEP

33. L3 Collab., O. Adriani et al., Phys. Lett. B ??? (1993) ???.

CERN preprint CERN-PPE/93-37; March 2, 1993.

Search for Narrow Vector Resonances in the Z Mass Range

34. L3 Collab., O. Adriani et al., Phys. Lett. B ??? (1993) ???.

CERN preprint CERN-PPE/93-44; March 8, 1993.

Search for a $\mathrm{Z}$ ' at the $\mathrm{Z}$ Resonance

35. Working Group on LEP Energy and the LEP Collaborations ALEPH, DELPHI, L3 and OPAL, L. Arnaudon et al., Phys. Lett. B ??? (1993) ???

CERN preprint CERN-PPE/93-53; March 16, 1993.

Measurement of the Mass of the Z Boson and the Energy Calibration of LEP

36. L3 Collab., O. Adriani et al., Phys. Lett. B ??? (1993) ???.

CERN preprint CERN-PPE/93-60; April 15, 1993.

Measurement of $\Gamma_{6 b} / \Gamma_{\text {had }}$ from Hadronic Deacys of the Z

\section{Doctoral Theses}

1. Q. Wang, "Measurement of the $\mathrm{Z}$ Decays to Muon Pairs With the L3 Detector at LEP," Thesis Submitted to Harvard University, January 1991.

2. I. Scott, "A Measurement of the Polarization of Tau Leptons in Z Decays with the L3 Detector at LEP," Thesis Submitted to Harvard University, May 1993. 


\section{Theory Group}

Paper:

The Quantum Mechanics of Trapped Wave Packets in Proceedings of the International School of Physics E. Fermi, Course CXVIII, E. Arimondo, W.D. Phillips, and F. Strumia, eds., North Holland, Amsterdam 1992, p. 643

Invited Papers on Quantum Theory of Particle Trapping:

1. Richard Brewer Symposium, Stanford University, May 16, 1992

2. Workshop on Squeezed Stated and Uncertainty Relations, Russian Academy of Sciences, Moscow, May 25, 1992

3. Conference on Physics of Quantum Electronics, Snowbird, Utah, January 5, 1993

4. International Workshop on Quantum Optics, Kühtai, Austria, March 9, 1993

\section{Colloquium Lectures on Quantum Theory of Particle Trapping:}

1. University of Auckland, New Zealand, Aug. 24, 1992

2. In Australia, Sep. 4-12, 1992:

- C.S.I.R.O. Laboratory, Melbourne

- Australian National University, Canberra

- University of Queensland, Brisbane

- Maquarie University, Sydney

3. University of Arizona, Tucson, Jan. 13, 1993

4. University of New Mexico, Albuquerque, Mar. 30, 1993

5. Theoretical Physics Division, Los Alamos National Laboratory, Apr. 1, 1993

\section{Doctoral Thesis:}

Wenyue Hsu, Quantum Electrodynamics in Resonant Cavities, Harvard University, May 1993 

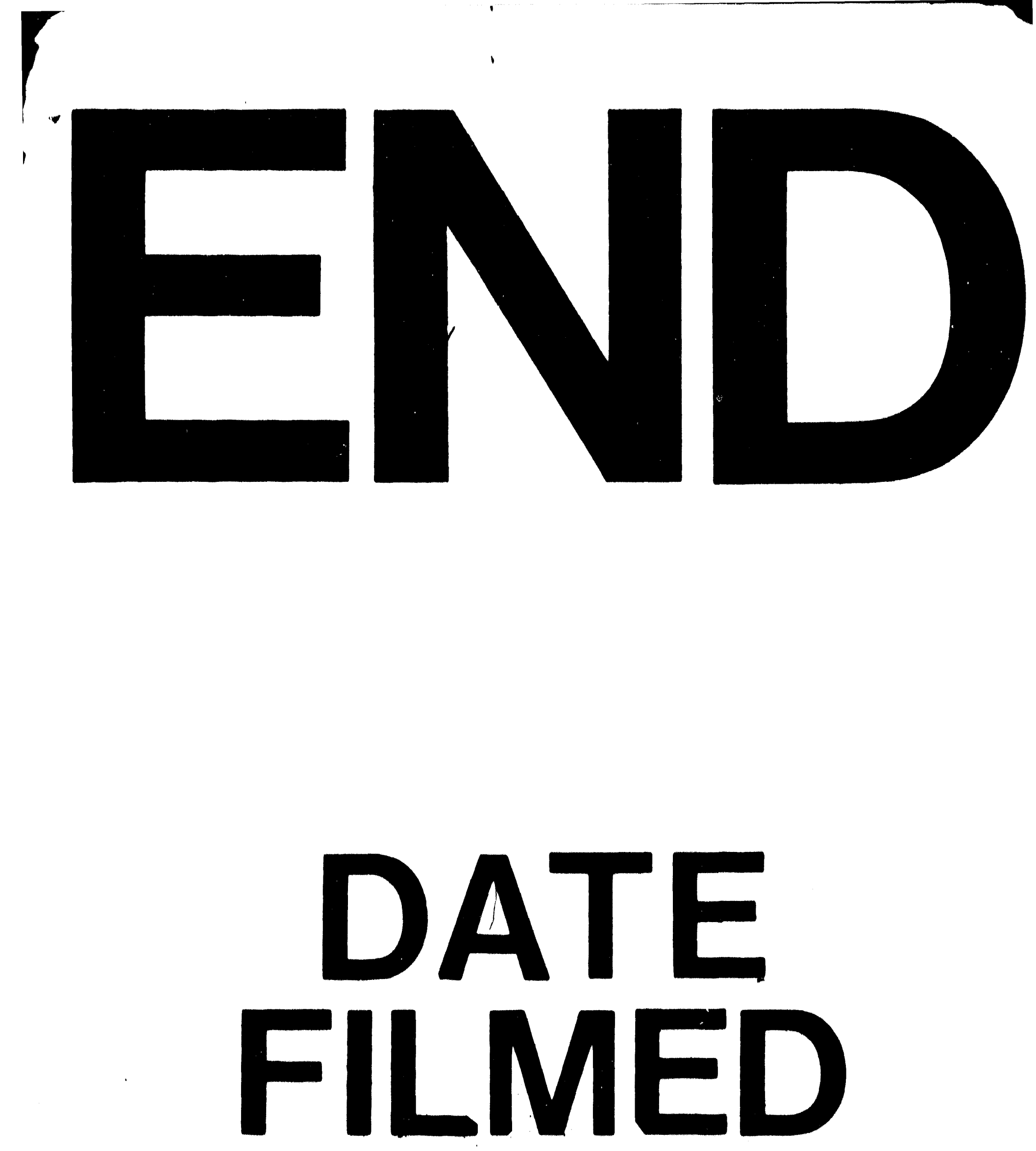

$$
1112194
$$




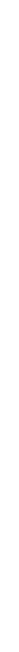

